Classification

Physics Abstracts

$05.30-\mathrm{d}$

\title{
Ursell Operators in Statistical Physics II: Microscopic Properties of a Dilute Quantum Gas
}

\author{
P. Grüter and F. Laloë \\ Laboratoire Kastler Brossel de l'ENS(*), France
}

(Received 7 April 1995, accepted 20 June 1995)

\begin{abstract}
We apply the formalism of Ursell operators, introduced in a previous article, to the calculation of reduced density operators (one and two-body density operators) in a dilute quantum gas; the calculation is not a fugacity expansion and is therefore not limited to low degrees of degeneracy. We obtain quantum corrections for the one-particle density operator as a function of the second Ursell operator. For the two-body density operator, we examine how statistics and interactions combine their effects on the correlations between particles; in particular we discuss in detail how hard cores potentials affect the short range correlations, a non-perturbative effect.
\end{abstract}

\section{Introduction}

In a previous article [1] (see also [2]), we introduced a method for obtaining the partition function of a degenerate gas which combines the use of cluster operators $U_{l}$, associated with an auxiliary system of distingurshable particles, with exchange cycles for an exact treatment of statistics. The basic motivation of this technique was to give a better treatment of correlations between particles than in mean field approaches, especially at short relative distances for strongly repulsive interaction potentials. In this article we show that the method is indeed a useful tool for obtaining explicit expressions of partial density operators, in particular of the two-particle density operators which, in princıple, contain all two-body correlations. The expressions remain valid at short distances even in the presence of hard core potentials, which means that they include physical effects occurring "in the middle of a collision" and not only asymptotic effects occurring long before or after collision (phase shifts).

In transport theory, an important issue is the derivation of kinetic equations, which is usually made by introducing appropriate approximations into the exact equations of motion of the system. A traditional approach is to start from the first equations of the BBGKY hierarchy and to use them to derive (or to guess) an approximate expression for the two-body density operator, which becomes a function of the one body density operator. The expression is then

(") This laboratory is an "Unité associée au CNRS UA-18 et à l'Université Pierre et Marıe Curıe", France 
inserted into the first BBGYK equation in order to obtain the desired kinetic equation (see for instance [3]). A well known example is the derivation given by Snider [4] of a quantum kinetic equation bearing his name for particles with or without internal states. The detailed study of the properties of reduced density operators may therefore be a useful point of comparison In this domain; for instance, one may require that the ansatz made for the expression of the two-body density operator (as a function of the one body operator) be valid at equilibrium, which provides a useful guide.

\section{Partial Density Operators}

In classical physics, the one-particle distribution function in a homogeneous system is independent of the interaction potential; this is because the contribution of the potential factorizes out of the distribution, which leaves in factor a Gaussian distribution function of the momentum. In quantum statistical mechanics, since the operators corresponding to kinetic and potential energy do not commute, this factorization does not take place so that the momentum distribution is no longer a Gaussian. One sometimes uses the words "diffraction effects" to characterize these changes (since they arise from the wave properties of particles), as opposed to "quantum statistics effects", which arise from the consequences of particle indistinguishability and also modify the distribution function. Our purpose in this section is to study how these two effects combine in the expression of the one and two particle density operators, in terms of the second Ursell operator $U_{2}$, of the ternary operator $U_{3}$, etc. We will use a technique based on the partial derivative of the operators, already sketched in [2], which starts from the following general expression of the canonical partition function $Z_{N}$ (the notation is the same as in [1]):

$$
Z_{N}=\operatorname{Tr}\left\{K_{N}^{S} A\right\}
$$

where the symmetrizer $S$ applies for bosons, the antisymmetrizer $A$ for fermions, and where $K_{N}$ is the exponential of the $N$ particle Hamiltonian $H_{N}$ multiplied by the inverse temperature $\beta$

$$
K_{N}=e^{-\beta H_{N}}
$$

which can be expanded as

$$
K_{N}=\sum_{\{U\}} \underbrace{U_{1}(.) U_{1}(.) \ldots U_{1}(.)}_{m_{1}^{\prime} \text { factors }} \times \underbrace{U_{2}(., .) U_{2}(., .) \ldots U_{2}(., .)}_{m_{2}^{\prime} \text { factors }} \times U_{3}(.,,,) .
$$

We recall the definitions of the lowest order Ursell operators:

$$
\begin{array}{cc}
U_{1}(1)= & \mathrm{e}^{-\beta H_{1}(1)} \\
U_{2}(1,2)= & \mathrm{e}^{-\beta H_{2}(1,2)}-U_{1}(1) \times U_{1}(2)
\end{array}
$$

By recurrence, higher orders are obtained in a similar way.

2.1. One-PARTICle DENSITy operator. - If we $\operatorname{set}\left({ }^{1}\right)$.

$$
U_{l}(1,2, . l)=\bar{U}_{l}(1,2, . l)\left[U_{1}(1) \times U_{1}(2) \times . \times U_{1}(l)\right]
$$

( $\left.{ }^{1}\right)$ With this definition, $\bar{U}_{l}$ is non-Hermitian; we could of course introduce only Hermitian operators by symmetrizing (5), namely by putting square roots of $U_{1}$ 's on both sides of $\bar{U}_{2}$, but this is not especrally useful for our calculations. 
we can choose $U_{1}$ and the $\bar{U}_{l}$ 's, instead of the $U_{l}$ 's, to characterize the cluster properties of $K_{N}$; by definition $\bar{U}_{1}$ is equal to one. Assume now that we keep all $\bar{U}_{l}$ 's constant and vary $U_{1}$ according to $\left({ }^{2}\right)$ :

$$
\mathrm{d} U_{1}=\mathrm{d} x \times U_{1}|\varphi\rangle\langle\theta| .
$$

What is then the variation of $Z$ ? Inserting this relation into (5), then into (3) and finally into (1), we get

$$
\mathrm{d} Z_{N}=\mathrm{d} x \operatorname{Tr}_{1,2,3, \ldots N}\left\{\begin{array}{l}
S \\
A
\end{array} K_{N} \sum_{i=1}^{i=N}|i: \varphi\rangle\langle i: \theta|\right\} .
$$

Since the canonical $N$ particle density operator is equal to

$$
\rho_{N}^{\text {can }}=\left[Z_{N}\right]^{-1}\left\{\begin{array}{ll}
S & S \\
A & K_{N}
\end{array}\right\}
$$

expression (7) is nothing but the product of $Z_{N}$ by the average value of the one-particle operator $\sum_{i=1}^{i=N}|i: \varphi\rangle\langle i: \theta|$. Therefore, in terms of the canonical one-particle density operator $\left(^{3}\right) \rho_{\mathrm{I}}^{\mathrm{can}}$, we obtain

$$
\frac{\mathrm{d}}{\mathrm{d} x} \log Z_{N}=\operatorname{Tr}\left\{|\varphi\rangle\langle\theta| \rho_{\mathrm{I}}^{\mathrm{can}}\right\}=\left\langle\theta\left|\rho_{\mathrm{I}}^{\mathrm{can}}\right| \varphi\right\rangle
$$

(the derivative with respect to $x$ is meant at the value $x=0$ ). We can therefore obtain reduced density matrices from this kind of operatorial variations.

If the grand canonical ensemble is used, the many-particle density operator is defined by

$$
\rho_{\mathrm{gc}}=\left[Z_{\mathrm{gc}}\right]^{-1} \sum_{N} e^{\beta \mu N}\left\{\begin{array}{l}
S \\
A
\end{array} K_{N}^{S}\right\}
$$

and a similar reasoning provides the following relation involving the grand canonical oneparticle density operator $\rho_{\mathrm{I}}$ (for the sake of simplicity we now omit the index $(\mathrm{gc})$ )

$$
\frac{\mathrm{d}}{\mathrm{d} x} \log Z_{\mathrm{gc}}=\operatorname{Tr}\left\{\rho_{\mathrm{I}}|\varphi\rangle\langle\theta|\right\}=\left\langle\theta\left|\rho_{\mathrm{I}}\right| \varphi\right\rangle
$$

This formula shows that $\rho_{\mathrm{I}}$ is the "operatorial derivative" of $Z_{\mathrm{gc}}$.

\subsection{Two-Particle Density Operator. - We now vary $U_{1}$ according to (see note 2)}

$$
\mathrm{d} U_{1}=U_{1}\left[\mathrm{~d} x\left|\varphi_{x}\right\rangle\left\langle\theta_{x}|+\mathrm{d} y| \varphi_{y}\right\rangle\left\langle\theta_{y}\right|\right]
$$

and we calculate the term, in the variation of $Z$, which is proportional to $\mathrm{d} x \times \mathrm{d} y$ (cross term). This term is given by

$$
\mathrm{d} x \times \mathrm{d} y \times \operatorname{Tr}_{1,2 \ldots N}\left\{\begin{array}{l}
S \\
{ }_{A}
\end{array} K_{N} \sum_{i \neq j}\left|i: \varphi_{x} ; j: \varphi_{y}\right\rangle\left\langle i: \theta_{x} ; j: \theta_{y}\right|\right\}
$$

$\left(^{2}\right)$ As $\bar{U}_{l}$, the variation $\mathrm{d} U_{1}$ is non Hermitian in general. Note that the $U_{1}$ 's are at the end of the product in (5) while they are at the beginning in (6); in fact, we could also choose the opposite convention where the $U_{1}$ 's are at the other end in both equations.

$\left({ }^{3}\right)$ Here we take the convention where the operator $\rho_{\mathrm{I}}$ is normalized to the number $N$ of particles (not to one). 
which is nothing but the product

$$
\mathrm{d} x \times \mathrm{d} y \times Z_{N} \times\left\langle\theta_{x}, \theta_{y}\left|\rho_{\mathrm{II}}^{\mathrm{can}}\right| \varphi_{x}, \varphi_{y}\right\rangle
$$

containing the matrix element of the two particle density operator $\left({ }^{4}\right) \rho_{\mathrm{II}}^{\text {can }}$ which is therefore given by

$$
\left\langle\theta_{x}, \theta_{y}\left|\rho_{\mathrm{II}}^{\mathrm{can}}\right| \varphi_{x}, \varphi_{y}\right\rangle=\left[Z_{N}\right]^{-1} \frac{\partial^{2}}{\partial x \partial y} Z_{N}
$$

Going over to the grand canonical ensemble leads to the relation

$$
\left\langle\theta_{x}, \theta_{y}\left|\rho_{\mathrm{II}}\right| \varphi_{x}, \varphi_{y}\right\rangle=\left[Z_{\mathrm{gc}}\right]^{-1} \frac{\partial^{2}}{\partial x \partial y} Z_{\mathrm{gc}}
$$

(where the derivative is meant in $x=y=0$ and, again, the index (gc) is not explicitly written for the reduced operator). But since

$$
\frac{\partial^{2}}{\partial x \partial y} \log Z_{\mathrm{gc}}=\left[Z_{\mathrm{gc}}\right]^{-1} \frac{\partial^{2} Z_{\mathrm{gc}}}{\partial x \partial y}-\left[Z_{\mathrm{gc}}\right]^{-2} \frac{\partial Z_{\mathrm{gc}}}{\partial x} \times \frac{\partial Z_{\mathrm{gc}}}{\partial y}
$$

we get, with the help of relation (11) (again, for simplification, we suppress the index (gc) from the reduced operator)

$$
\left\langle\theta_{x}, \theta_{y}\left|\rho_{\mathrm{II}}\right| \varphi_{x} \varphi_{y}\right\rangle=\left\langle\theta_{x}\left|\rho_{\mathrm{I}}\right| \varphi_{x}\right\rangle\left\langle\theta_{y}\left|\rho_{\mathrm{I}}\right| \varphi_{y}\right\rangle+\frac{\partial^{2}}{\partial x \partial y} \log Z_{\mathrm{gc}}
$$

This is the starting point of our calculations below.

\section{Application to a Dilute Gas}

In [1] we obtained expressions for $\log Z$ that are valid for dilute gases. We now combine them with the preceding formulas to obtain reduced density operators, starting from the simple example of an ideal gas (no interactions).

3.1. IDEAL GaS. - For an ideal gas we have

$$
\log Z=-\eta \operatorname{Tr}\left\{\log \left[1-\eta z U_{1}\right]\right\}=\eta \sum_{n=1}^{\infty} n^{-1} \operatorname{Tr}\left\{\left[\eta z U_{1}\right]^{n}\right\}
$$

where $\eta$ is equal to 1 for bosons, -1 for fermions, and

$$
z=\mathrm{e}^{\beta \mu}
$$

3.1.1. One-Particle Density Operator. - When $U_{1}$ varies according to $(6)$ in the right hand side of (19), we have to vary the $n$ factors of the product $\left[\eta z U_{1}\right]^{n} \quad$ But, in fact, all these terms are equal since, in each of them, a permutation of the operators under the trace allows us to bring the variation in front of all the other operators; the factor $n^{-1}$ disappears in this operation and the result is

$$
\mathrm{d} \log Z=\eta \mathrm{d} x \times \sum_{n=1}^{\infty} \operatorname{Tr}\left(\eta z U_{1}|\varphi\rangle\langle\theta|\left[\eta z U_{1}\right]^{n-1}\right)
$$

$\left({ }^{4}\right)$ with a normalization to $(N-1) N$. 
or

$$
\frac{\mathrm{dLog} Z}{\mathrm{~d} x}=\eta\left\langle\theta\left|\sum_{n=1}^{\infty} \operatorname{Tr}\left\{\left[\eta z U_{1}\right]^{n}\right\}\right| \varphi\right\rangle=\left\langle\theta\left|\rho_{\mathrm{I}}\right| \varphi\right\rangle .
$$

This provides the simple result

$$
\rho_{\mathrm{I}}=f
$$

where the operator $f$ is defined as a function of the operator $U_{1}$ as

$$
f=\frac{z U_{1}}{1-\eta z U_{1}}
$$

or, more explicitly (in the case where the external potential is zero), as

$$
f=\frac{\mathrm{e}^{-\beta\left[\frac{\mathrm{P}^{2}}{2 m}-\mu\right]}}{1-\eta \mathrm{e}^{-\beta\left[\frac{\mathrm{P}^{2}}{2 m}-\mu\right]}}
$$

(this is nothing but the operatorial version of the well known Fermi of Bose functions for an ideal quantum gas). We therefore recover a classical result.

3.1.2. Two Particle Density Operator. - We now vary $U_{1}$ in (19) according to (12) and look for the cross terms in $\mathrm{d} x$ and $\mathrm{d} y$. As in the preceding section, in every term of the sum over $n$ the replacement by $\mathrm{d} x\left|\varphi_{x}\right\rangle\left\langle\theta_{x}\right| U_{1}$ can occur in $n$ different places, but can be brought back to the front position by circular permutation under the trace, which suppresses the factor $n^{-1}$ The cross term appearing in $\eta n^{-1} \operatorname{Tr}\left\{\left[\eta z U_{1}\right]^{n}\right\}$ is therefore equal to

$$
\eta \mathrm{d} x \mathrm{~d} y \operatorname{Tr}\left\{\eta z U_{1}\left|\varphi_{x}\right\rangle\left\langle\theta_{x}\left|\sum_{p=0}^{n-2}\left[\eta z U_{1}\right]^{p} \eta z U_{1}\right| \varphi_{y}\right\rangle\left\langle\theta_{y}\right|\left[\eta z U_{1}\right]^{n-2-p}\right\}
$$

which, when summed over $n$, introduces the quantity:

$$
\eta \sum_{n=1}^{\infty} \sum_{p=0}^{n-2}\left\langle\theta_{x}\left|\left[\eta z U_{1}\right]^{p+1}\right| \varphi_{y}\right\rangle\left\langle\theta_{y}\left|\left[\eta z U_{1}\right]^{n-1-p}\right| \varphi_{x}\right\rangle
$$

We can set $p^{\prime}=n-p-2$ and replace the summations over $n$ and $p$ by summations over $n$ and $p^{\prime}$; then, each of these summations introduces again the operator $f$ defined in (24). Inserting this into the right hand side of (18), we get

$$
\left\langle\theta_{x}, \theta_{y}\left|\rho_{\mathrm{II}}\right| \varphi_{x}, \varphi_{y}\right\rangle=\left\langle\theta_{x}|f| \varphi_{x}\right\rangle \times\left\langle\theta_{y}|f| \varphi_{y}\right\rangle+\eta\left\langle\theta_{x}|f| \varphi_{y}\right\rangle \times\left\langle\theta_{y}|f| \varphi_{x}\right\rangle
$$

or

$$
\rho_{\mathrm{II}}(1,2)=[f(1) \otimes f(2)]\left[1+\eta P_{\mathrm{ex}}\right]
$$

where $f(1)$ is the $f$ operator with the momentum of the first particle inside $U_{1}$ :

$$
f(1)=\frac{z U_{1}(1)}{1-\eta z U_{1}(1)}
$$

while $f(2)$ has the same definition for the other particle; $P_{\text {ex }}$ is the exchange operator between particles 1 and 2. Equation (29) is of course a known result for ideal gases. 
Remark: from (23) and (29), since the trace of the one-particle density operator is equal to the average number of particles, we obtain

$$
\operatorname{Tr}_{2}\left\{\rho_{\mathrm{II}}(1,2)\right\}=<N>\rho_{\mathrm{I}}(1)+\eta\left[\rho_{\mathrm{I}}(1)\right]^{2}
$$

which shows that the partial trace of the two-body density operator is not merely proportional to the one-particle density operator, but contains an additional term proportional to $\left[\rho_{\mathrm{I}}(1)\right]^{2}$ This is a consequence of the use of the grand canonical ensemble where the number of particles $N$ fluctuates, so that for instance the average of the product $N(N-1)$ is not exactly equal to $\langle N\rangle\langle N-1\rangle$. In the thermodynamic limit, nevertheless, where the volume and $\langle N\rangle$ tend simultaneously to infinity (while their ratio remains constant), since the expression of the operator $\rho_{\mathrm{I}}$ itself does not depend on the volume, it is clear that the first term in (31) 1s dominant, so that the usual trace relation of the canonical ensemble

$$
\operatorname{Tr}_{2}\left\{\rho_{\mathrm{II}}^{\mathrm{can}}(1,2)\right\}=<N-1>\rho_{\mathrm{I}}^{\mathrm{can}}(1)
$$

is recovered to a very good approximation. Physically, this happens because the exchange term in (29) is significant at microscopic relative distances between the particles, but vanishes when this distance becomes macroscopic; therefore, by average over all possible positions of the second particle, the direct term becomes dominant.

3.2. INTERACTING DILUTE GAS: FIRST CORRECTION. - Our purpose now is to introduce interactions and to examine how (23) and (29) are modified. We shall start from the simplest generalization $\left({ }^{5}\right)$ of the Beth Uhlenbeck formula introduced in [1]

$$
\begin{aligned}
\log Z & =[\log Z]_{\mathrm{ig}}+z^{2} \operatorname{Tr}_{1,2}\left\{U_{2} \frac{1+\eta P_{\mathrm{ex}}}{2} \times[1+\eta f(1)] \times[1+\eta f(2)]\right\} \\
& =[\log Z]_{\mathrm{ig}}+\operatorname{Tr}_{1,2}\left\{\bar{U}_{2} \frac{1+\eta P_{\mathrm{ex} .}}{2} \times f(1) \times f(2)\right\}
\end{aligned}
$$

where $[\log Z]_{\mathrm{ig}}$ is the value obtained in the absence of interactions, given in (19); according to (5), the operator $\bar{U}_{2}$ is defined by

$$
\bar{U}_{2}=U_{2} \times\left[U_{1}(1) U_{1}(2)\right]^{-1}
$$

3.2.1. One-Particle Density Operator. - The product $f(1) f(2)$ in the second line of (33) can be expanded according to

$$
f(1) f(2)=\sum_{n, p=1}^{\infty}\left[\eta z U_{1}(1)\right]^{n} \times\left[\eta z U_{1}(2)\right]^{p}
$$

(we use the fact that $\eta^{2}=1$ for bosons and fermions) so that the variations of $U_{1}$ defined in (6) introduce a term equal to

$$
\begin{array}{r}
\operatorname{Tr}_{1,2}\left\{\bar{U}_{2} \frac{1+\eta P_{\text {ex. }}}{2} \sum_{n=1}^{\infty} \sum_{k=0}^{n-1}\left[\eta z U_{1}(1)\right]^{k} \eta z U_{1}(1)|1: \varphi><1: \theta|\right. \\
\left.\left[\eta z U_{1}(1)\right]^{n-1-k} \eta f(2)\right\}+ \text { id. }(1 \leftrightarrow 2)
\end{array}
$$

$\left({ }^{5}\right)$ In the second line of (33), the factor $z^{2}$ appearing in the right hand side of the first line has been combined with the $U_{1}$ 's introduced by the substitution of $\bar{U}_{2}$ for $U_{2}$ in order to provide the two operators $f$ 's. 
where id. $(1 \leftrightarrow 2)$ symbolizes the same expression with indices 1 and 2 exchanged. The summation over $n$ can be replaced by a summation over $n^{\prime}=n-1-k$ which introduces the operator

$$
\sum_{n^{\prime}=0}^{\infty}\left[\eta z U_{1}(1)\right]^{n^{\prime}}=1+\eta f(1)
$$

while the summation over $k$ introduces

$$
\sum_{k=0}^{\infty}\left[\eta z U_{1}(1)\right]^{k+1}=\frac{\eta z U_{1}(1)}{1-\eta z U_{1}(1)}=\eta f(1) .
$$

Now, since

$$
f(1)=z U_{1}(1)[1+\eta f(1)]
$$

the factor $\eta f(2)$ in the right hand side of (36) and the $\eta f(1)$ from (38) can be inserted into $\bar{U}_{2}$ to provide the result $z^{2} U_{2}[1+\eta f(1)][1+\eta f(2)]$, which leads to

$$
2 z^{2}\left\langle\theta\left|[1+\eta f(1)] \operatorname{Tr}_{2}\left\{U_{2} \frac{1+\eta P_{\text {ex. }}}{2}[1+\eta f(1)][1+\eta f(2)]\right\}\right| \varphi\right\rangle
$$

(the factor 2 arises from the term $[1 \leftrightarrow 2]$ ). Finally we obtain

$$
\rho_{\mathrm{I}}=f(1)+2 z^{2}[1+\eta f(1)] \operatorname{Tr}_{2}\left\{U_{2} \frac{1+\eta P_{\mathrm{ex}}}{2}[1+\eta f(2)]\right\}[1+\eta f(1)]
$$

This equation is valid to first-order in $U_{2}$.

The validity of (40) does not require that the degree of degeneracy of the gas should be very low, but of course it can be applied to this particular case: in the limit of a non-degenerate gas, the correction to $\rho_{\mathrm{I}}$ is simply obtained by replacing in (40) all factors $[1+\eta f]$ by one. One can also expand these factors in powers of $\eta f$, which provides the quantum corrections introduced by particle indistinguishability, starting with the term corresponding to a permutation between two particles, followed by the term introduced by cycles of three particles, etc.

Equation (40) is limited to interaction potentials with sufficiently small range $b$, a necessary conditions for the higher order corrections in $\left[U_{2}\right]^{2},\left[U_{2}\right]^{3}$, etc. as well in any power of $U_{3}, U_{4}$, etc. to be negligible $\left({ }^{6}\right)$; on the other hand, it is not a perturbative result with respect to the intensity of the interaction potential, which can take arbitrarily large values for small relative distances between particles (see for instance Section 4 for a discussion of hard core potentials); in other words, the equation contains the effects of local perturbations on thermal equilibrium, even if they are strong.

Mathematically, $U_{2}$ is the difference between two exponential operators and, as such, contains all powers of the potential. In order to make contact with perturbation theories, it is of course possible to retain only the first-order term from the $V$ expansion of $U_{2}$, that is to replace $U_{2}(1,2)$ by

$$
U_{2} \simeq-\int_{0}^{\beta} \mathrm{d} \beta^{\prime} U_{1}^{\beta-\beta^{\prime}}(1) U_{1}^{\beta-\beta^{\prime}}(2) V(1,2) U_{1}^{\beta^{\prime}}(1) U_{1}^{\beta^{\prime}}(2)
$$

where

$$
U_{1}^{\beta^{\prime \prime}}(1)=\mathrm{e}^{-\beta^{\prime \prime}\left[\mathbf{P}^{2} / 2 m+V_{\text {ext }}(\mathbf{r})\right]}
$$

$\left({ }^{6}\right)$ See Section 3.3 for a discussion of these terms. 
and where $V_{\text {ext }}(\mathbf{r})$ is the external potential. This yields

$$
\rho_{\mathrm{I}} \simeq f(1)+2 f(1) \int_{0}^{\beta} \mathrm{d} \beta^{\prime} U_{1}^{\beta^{\prime}}(1) \operatorname{Tr}_{2}\left\{V(1,2) \frac{1+\eta P_{\text {ex. }}}{2} f(2)\right\} U_{1}^{\beta^{\prime}}(1)[1+\eta f(1)]
$$

an equation which now requires the potential to be sufficiently small. In the case of fermions $(\eta=-1)$ the diagonal element of $\rho_{\text {I }}$ gets a non-zero correction only if the product $f(1)[1-f(1)]$ is non zero, where $[1-f(1)]$ can be interpreted as the distribution function of holes; as a consequence, the correction occurs only near the surface of the Fermi sphere, within an energy range of the order of $k T$, which is a familiar result $\left({ }^{7}\right)$. The fact that (43) contains integral over the inverse temperature $\beta^{\prime}$ is characteristic of the results of perturbation theories $[5,6]$ (second order terms would contain a double integration over $\beta^{\prime}$ and $\beta^{\prime \prime}$, etc.), as opposed to our formalism where every $U_{2}$ contains a resummation of an infinite perturbation series which suppresses these summations.

3.2.2. Two-Particle Density Operator. - We now insert (12) into (35) and look for the crossed terms in $\mathrm{d} x$ and $\mathrm{d} y$. Several possibilities may arise, depending on whether the variations affect two $U_{1}$ 's corresponding to two different particles, or to the same particle. This introduces under the double sum over $n$ and $p$ the following expression

$$
\begin{gathered}
\sum_{k=0}^{n-1}\left[\eta z U_{1}(1)\right]^{k} \eta z U_{1}(1)\left|1: \varphi_{x}\right\rangle\left\langle 1: \theta_{x}\right|\left[\eta z U_{1}(1)\right]^{n-1-k} \times \\
\times \sum_{k^{\prime}=0}^{p-1}\left[\eta z U_{1}(2)\right]^{k^{\prime}} \eta z U_{1}(2)\left|2: \varphi_{y}\right\rangle\left\langle 2: \theta_{y}\right|\left[\eta z U_{1}(2)\right]^{p-1-k^{\prime}}+ \\
+\sum_{k=0}^{n-2}\left[\eta z U_{1}(1)\right]^{k} \eta z U_{1}(1)\left|1: \varphi_{x}\right\rangle\left\langle 1: \theta_{x}\right| \sum_{k^{\prime}=0}^{n-2-k}\left[\eta z U_{1}(1)\right]^{k^{\prime}} \\
\eta z U_{1}(1)\left|1: \varphi_{y}\right\rangle\left\langle 1: \theta_{y}\right|\left[\eta z U_{1}(1)\right]^{n-2-k-k^{\prime}}\left[\eta z U_{1}(2)\right]^{p} \\
+\operatorname{id} .([1, n] \leftrightarrow[2, p])+\mathrm{id} .(x \leftrightarrow y)
\end{gathered}
$$

where, in the last line, id. $([1, n] \leftrightarrow[2, p])$ orlginates from two variations in $U_{1}(2)$ 's, which introduce a term equal to that contained in the two preceding lines with the substitutions indicated between brackets. Moreover, since the first operator which is varied can contain a variation in $\left|\varphi_{x}\right\rangle\left\langle\theta_{x}\right|$ as assumed explicitly in (44), or a variation in $\left|\varphi_{y}\right\rangle\left\langle\theta_{y}\right|$, one should add to all preceding results the same term with the substitution $(x \leftrightarrow y)$, as indicated in the second term of the last line. The rest of the calculation is simlar to that of the preceding section: when independent summation indices replace $n, p, k$ and $k^{\prime}$, the summations introduce either operators $\eta f$ 's (when the index runs from 1 to infinity) or operators $[1+\eta f]$ (when it runs from 0 to infinity) so that (44) becomes

$$
\begin{gathered}
\eta f(1)\left|1: \varphi_{x}\right\rangle\left\langle 1: \theta_{x}|[1+\eta f(1)] \eta f(2)| 2: \varphi_{y}\right\rangle\left\langle 2: \theta_{y}\right|[1+\eta f(2)]+ \\
+\eta f(1)\left|1: \varphi_{x}\right\rangle\left\langle 1: \theta_{x}|\eta f(1)| 1: \varphi_{y}\right\rangle\left\langle 1: \theta_{y}\right|[1+\eta f(1)] \eta f(2) \\
+ \text { id. }(1 \leftrightarrow 2)+\operatorname{id.}(x \leftrightarrow y)
\end{gathered}
$$

According to (33), we now have to multiply this term by $\bar{U}_{2}$ and a symmetrization operator, and to take the trace over the variables of two particles. As in the calculation of $\rho_{\mathrm{I}}$, it is

$\left({ }^{7}\right)$ This remains true if the more general formula (40) is used, as can be seen by replacing $U_{2}[1+\eta f(1)][1+\eta f(2)]$ by the product $\bar{U}_{2} f(1) f(2)$. 
convenient to move $\eta f(1)$ as well as $\eta f(2)$ towards $\bar{U}_{2}$, with which they combine to introduce the product $z^{2} U_{2}[1+\eta f(1)][1+\eta f(2)]$; doing this operation, for instance, in the term of the first line of $(45)$ provides the result

$$
\begin{aligned}
z^{2} \operatorname{Tr}_{1,2}\left\{U_{2}^{\mathrm{S}, \mathrm{A}}\right. & {[1+\eta f(1)]\left|1: \varphi_{x}\right\rangle\left\langle 1: \theta_{x}\right|[1+\eta f(1)] \times } \\
& \left.\times[1+\eta f(2)]\left|2: \varphi_{y}\right\rangle\left\langle 2: \theta_{y}\right|[1+\eta f(2)]\right\}
\end{aligned}
$$

which is simply equal to

$$
z^{2}\left\langle\theta_{x}, \theta_{y}\left|[1+\eta f(1)][1+\eta f(2)] U_{2}^{\mathrm{S}, \mathrm{A}}[1+\eta f(1)][1+\eta f(2)]\right| \varphi_{x}, \varphi_{y}\right\rangle
$$

where we have introduced the notation $U_{2}^{\mathrm{S}, \mathrm{A}}$ for the restriction of $U_{2}$ to the symmetric/antisymmetric space:

$$
U_{2}^{\mathrm{S}, \mathrm{A}}=U_{2} \frac{1+\eta P_{\mathrm{ex}}}{2}
$$

(because the operator $U_{2}$ itself is already symmetric by exchange of the particles, in this definition the symmetrization projector $\left[1+\eta P_{\text {ex. }}\right] / 2$ may arbitrarly be put on either side, or on both). Similarly, the second line of (45) introduces a contribution

$$
z^{2}\left\langle\theta_{x}|\eta f(1)| \varphi_{y}\right\rangle\left\langle\theta_{y}\left|[1+\eta f(1)] \operatorname{Tr}_{2}\left\{U_{2}^{\mathrm{S}, \mathrm{A}}[1+\eta f(2)]\right\}[1+\eta f(1)]\right| \varphi_{x}\right\rangle
$$

This can be written as a term containing a trace over a third particle

$$
\begin{aligned}
& z^{2}\left\langle\theta_{x}, \theta_{y}\right|[1+\eta f(1)][1+\eta f(2)] \operatorname{Tr}_{3}\left\{U_{2}^{\mathrm{S}, \mathrm{A}}(2,3)[1+\eta f(3)]\right\} \times \\
& \times[1+\eta f(2)] \eta P_{\text {ex. }}(1,2)\left|\varphi_{x}, \varphi_{y}\right\rangle
\end{aligned}
$$

which, according to (40) and (18), is nothing but an exchange term associated with the product of $\rho_{\mathrm{I}}$ 's appearing in (18). Finally, if we include the contributions of the permutations in the third line of (45), our results concerning $\rho_{\mathrm{I}}$ and $\rho_{\mathrm{II}}$ can be summarized in the following equations, where we use the notation $1 \times U_{2}$ as a reminder that the present calculation is first-order in $U_{2}$

$$
\begin{gathered}
\rho_{\mathrm{I}}(1)=f(1)+\delta \rho_{\mathrm{I}}(1) \\
\rho_{\mathrm{II}}(1,2)=f(1) \otimes f(2)\left[1+\eta P_{\mathrm{ex}}\right]+\delta \rho_{\mathrm{II}}(1,2)
\end{gathered}
$$

with

$$
\delta \rho_{\mathrm{I}}=\delta f \equiv 2 z^{2}[1+\eta f(1)] \operatorname{Tr}_{2}\left\{U_{2}^{\mathrm{S}, \mathrm{A}}[1+\eta f(2)]\right\}[1+\eta f(1)]
$$

and

$$
\begin{gathered}
\delta \rho_{\mathrm{II}}=2 z^{2}[1+\eta f(1)][1+\eta f(2)] U_{2}^{\mathrm{S}, \mathrm{A}}(1,2)[1+\eta f(1)][1+\eta f(2)] \\
+\left[1+\eta P_{\mathrm{ex} .}\right]\left[f(1) \otimes \delta \rho_{I}(2)\right]\left[1+\eta P_{\mathrm{ex} .}\right]
\end{gathered}
$$

The latter result includes two kinds of contributions to $\delta \rho_{I I}$. In the second line, we get a correction as a direct consequence of the modification of $\rho_{\mathrm{I}}$ under the effects of the interactions, but which contains no correlation between the particles, except of course the statistical correlation introduced by the operators $\left[1+\eta P_{\text {ex. }}\right]$ at each end. In the first line, we get a different kind of correction which may contain strong short range correlations created by the interactions, that is correlations that manifest themselves over a characteristic distance of the order of the potential range $b$ (and not only of the order of the de Broglie wavelength). In Section 4 we discuss these short range effects in more detail.

Otherwise, the remarks made after equation (40) apply without any change. For instance, (53) is a non-perturbative result in terms of the intensity of the interactions, but is valid only if the potential range $b$ is sufficiently small. The limit of non-degenerate gases is immediately obtained by suppressing all brackets $[1+\eta f]$ in (52) and (53). 
3.3. SECOND-ORDER IN $U_{2}$. - We now include the effect of the terms in $\log Z$ which are second order in $U_{2}$ and have been obtained in [1]. Their contribution has been found to be equal to the sum of two terms

$$
\frac{z^{4}}{2} \operatorname{Tr}_{1,2}\left\{\left[U_{2}^{\mathrm{S}, \mathrm{A}}(1,2)[1+\eta f(1)][1+\eta f(2)]\right]^{2}\right\}
$$

and

$$
2 \eta z^{4} \operatorname{Tr}_{1,2,3}\left\{U_{2}^{\mathrm{S}, \mathrm{A}}(1,2)[1+\eta f(1)][1+\eta f(2)] U_{2}^{\mathrm{S}, \mathrm{A}}(1,3)[1+\eta f(1)][1+\eta f(3)]\right\} .
$$

Introducing the notation

$$
\bar{U}_{2}^{\mathrm{S}, \mathrm{A}}(1,2)=\bar{U}_{2}(1,2) \frac{1+\eta P_{\mathrm{ex}}}{2}
$$

we can rewrite (54) as

$$
\frac{1}{2} \operatorname{Tr}_{1,2}\left\{\bar{U}_{2}^{\mathrm{S}, \mathrm{A}}(1,2) f(1) f(2) \bar{U}_{2}^{\mathrm{S}, \mathrm{A}}(1,2) f(1) f(2)\right\} .
$$

From now on, we focus the calculation to the two particle density operator, which contains the correlations between the particles and therefore the most interesting physical information. The f's may be expanded in $U_{1}$ 's as in (35); when the $U_{1}$ 's are varied, several kinds of situations may occur, depending on whether the variations occur in close $f$ 's or $f$ 's separated by a $\bar{U}_{2}^{\mathrm{S}, \mathrm{A}}$, and whether the $f$ 's correspond to the same particle or not. Since all $f$ 's play the same role, we can assume that the variation in $\mathrm{d} x$ takes place in the first $f(1)$, and multiply the result by 4. There are now four possible cases, depending on where the second variation is placed.

1) If the second variation occurs in the same $f(1)$, the term which appears is similar to that calculated in the third and fourth lines of (44), and we have to make the substitution

$$
f(1) \Rightarrow \eta f(1)\left|1: \varphi_{x}\right\rangle\left\langle 1: \theta_{x}|f(1)| 1: \varphi_{y}\right\rangle\left\langle 1: \theta_{y}\right|[1+\eta f(1)]+\text { id. }(x \leftrightarrow y) .
$$

This leads to

$$
\begin{aligned}
2 \eta\left\langle\theta_{x}|f(1)| \varphi_{y}\right\rangle \times \operatorname{Tr}_{1,2}\left\{\bar{U}_{2}^{S, A}(1,2) f(1)\left|1: \varphi_{x}\right\rangle\left\langle 1: \theta_{y}\right| \times\right. \\
\left.\times[1+\eta f(1)] f(2) \bar{U}_{2}^{S, A}(1,2) f(1) f(2)\right\}
\end{aligned}
$$

But we also have to add the symmetric term id. $(x \leftrightarrow y)$, so that we get the following contribution to the two particle operator

$$
2 z^{4} f(1)[1+\eta f(2)] \operatorname{Tr}_{3}\left\{\left[U_{2}^{\mathrm{S}, \mathrm{A}}(2,3)[1+\eta f(2)][1+\eta f(3)]\right]^{2}\right\} \eta P_{\mathrm{ex}}(1,2)+(1 \leftrightarrow 2)
$$

where the term $(1 \leftrightarrow 2)$ is simply obtained from the preceding term by moving the exchange operator $P_{\mathrm{ex}}(1,2)$ in front of the other operators. The contribution $(60)$ is clearly an exchange term, which contains no dynamic correlation between the particles, but just correlations from statistics.

ii) If the variation occurs in the $f(2)$ which follows immediately the varied $f(1)$, both $f$ 's should be varied according to

$$
f \Rightarrow f\left|1: \varphi_{x, y}\right\rangle\left\langle 1: \theta_{x, y}\right|[1+\eta f]
$$


so that we obtain

$$
\begin{aligned}
2 \operatorname{Tr}_{1,2} & \left\{\bar{U}_{2}^{\mathrm{S}, \mathrm{A}}(1,2) f(1)\left|1: \varphi_{x}\right\rangle\left\langle 1: \theta_{x}\right|[1+\eta f(1)] f(2) \times\right. \\
& \left.\times\left|2: \varphi_{y}\right\rangle\left\langle 2: \theta_{y}\right|[1+\eta f(2)] \bar{U}_{2}^{\mathrm{S}, \mathrm{A}}(1,2) f(1) f(2)\right\}
\end{aligned}
$$

which leads to the following contribution

$$
\begin{aligned}
2 z^{4} & {[1+\eta f(1)][1+\eta f(2)] U_{2}^{\mathrm{S}, \mathrm{A}}(1,2)[1+\eta f(1)] \times } \\
& \times[1+\eta f(2)] U_{2}^{S, \mathrm{~A}}(1,2)[1+\eta f(1)][1+\eta f(2)] .
\end{aligned}
$$

This is a second-order generalization of the first line of (53), which contains no trace over a third particle; we expect that it will contain short range correlations for strongly repulsive potentials at short distances.

iii) If the second variation occurs in the other $f(1)$, equation (61) applies again and we obtain

$$
\begin{aligned}
2 \operatorname{Tr}_{1,2} & \left\{\bar{U}_{2}^{\mathrm{S}, \mathrm{A}}(1,2) f(1)\left|1: \varphi_{x}\right\rangle\left\langle 1: \theta_{x}\right|[1+\eta f(1)] f(2) \times\right. \\
& \left.\times \bar{U}_{2}^{\mathrm{S}, \mathrm{A}}(1,2) f(1)\left|1: \varphi_{y}\right\rangle\left\langle 1: \theta_{y}\right|[1+\eta f(1)] f(2)\right\}
\end{aligned}
$$

and the following contribution to the two particle density operator

$$
\begin{aligned}
& 2 z^{4}[1+\eta f(1)][1+\eta f(2)] \operatorname{Tr}_{3}\left\{U_{2}^{\mathrm{S}, \mathrm{A}}(1,3)[1+\eta f(1)][1+\eta f(3)] \times\right. \\
&\left.\times U_{2}^{\mathrm{S}, \mathrm{A}}(2,3)[1+\eta f(2)][1+\eta f(3)]\right\} P_{\mathrm{ex}}(1,2)
\end{aligned}
$$

The curious feature of this term is that it contains an exchange operator without a factor $\eta$; we will see other similar cases below.

iv) Finally, the variation may occur in the second $f(2)$, which from (61) leads to:

$$
\begin{aligned}
2 \operatorname{Tr}_{1,2} & \left\{\bar{U}_{2}^{\mathrm{S}, \mathrm{A}}(1,2) f(1)\left|1: \varphi_{x}\right\rangle\left\langle 1: \theta_{x}\right|[1+\eta f(1)] f(2) \times\right. \\
& \left.\times \bar{U}_{2}^{\mathrm{S}, \mathrm{A}}(1,2) f(1) f(2)\left|2: \varphi_{y}\right\rangle\left\langle 2: \theta_{y}\right|[1+\eta f(2)]\right\}
\end{aligned}
$$

which gives the same contribution to $\rho_{\mathrm{II}}$ as the term (65).

Among all four terms that we have obtained, only (63) contains dynamical correlations between the particles, and no trace over a third interaction partner. This term will play an important role in the discussion of Section 4 .

We now have to apply the same kind of calculation to (55). The general method is exactly the same, but here we obtain 5 terms instead of 4: one term which contains a trace over a third particle with, inside the trace, only one $U_{2}$ operator; one term of the same kind but with two $U_{2}$ 's inside the trace; and finally three terms containing traces over two extra particles. As an example, we give one of these three terms, which is equal to

$$
\begin{array}{r}
4 z^{4} f(1)[1+\eta f(2)] \operatorname{Tr}_{3,4}\left\{U_{2}^{\mathrm{S}, \mathrm{A}}(2,3)[1+\eta f(2)][1+\eta f(3)] \times\right. \\
\left.\times U_{2}^{\mathrm{S}, \mathrm{A}}(2,4)[1+\eta f(2)][1+\eta f(4)] P_{\mathrm{ex}}(1,2)\right\}+ \\
+ \text { id. }[1 \leftrightarrow 2] .
\end{array}
$$

To save space, we do not write the other terms explicitly.

Finally, one must not forget to include the second order modifications of the product of $\rho_{\mathrm{I}} \otimes \rho_{\mathrm{I}}$ according to $(18)$. They combine with the terms in $\eta P_{\mathrm{ex}}(1,2)$ of $(60)$ and the analog contribution derived from (55) in order to produce a symmetrizer/antisymmetrizer $\left[1+\eta P_{\mathrm{ex}}(1,2)\right] / 2$ in the same manner as for the first-order calculation. 
3.4. First-ORDER IN $U_{3}$. - In reference [1], we obtain the following term for the first-order contribution of $U_{3}$ to $\log Z$

$$
z^{3} \operatorname{Tr}_{1,2,3}\left\{U_{3}^{\mathrm{S}, \mathrm{A}}(1,2,3)[1+\eta f(1)][1+\eta f(2)][1+\eta f(3)]\right\}
$$

where $U_{3}^{\mathrm{S}, \mathrm{A}}(1,2,3)$ is the properly symmetrized version of the three particle operator $U_{3}$

$$
U_{3}^{\mathrm{S}, \mathrm{A}}(1,2,3)=U_{3}(1,2,3) \frac{\mathrm{S}_{3}}{\mathrm{~A}_{3}} .
$$

It is convenient to rewrite (68) in the form

$$
\operatorname{Tr}_{1,2,3}\left\{\bar{U}_{3}^{\mathrm{S}, \mathrm{A}}(1,2,3) \times f(1) \times f(2) \times f(3)\right\}
$$

and, as in (35), to expand each of the $f$ 's inside the product into a series of $U_{1}$ 's which will be varied. If both variations occur in $U_{1}$ 's originating from the same $f$, we use (58) and we are led to the following contribution to $\rho_{\mathrm{II}}$ :

$$
3 f(1) \otimes[1+\eta f(2)] \operatorname{Tr}_{3,4}\left\{U_{3}(2,3,4)[1+\eta f(3)][1+\eta f(4)]\right\}[1+\eta f(2)] \eta P_{\text {ex. }}+\text { h.c. }
$$

where (h.c.) symbolizes the Hermitian conjugate operator, with $P_{\text {ex. }}$ in front of the product of operators instead of at the end. This term is similar to (60) and reminiscent of the exchange terms in $\rho_{\mathrm{II}}$ for an ideal gas.

If the variations of the $U_{1}$ occur in operators originating from two different $f$ 's, we use (61) and we are led to the following contribution to the two particle density operator

$$
6 z^{3}[1+\eta f(1)][1+\eta f(2)] \times \operatorname{Tr}_{3}\left\{U_{3}(1,2,3)[1+\eta f(3)]\right\} \times[1+\eta f(1)][1+\eta f(2)]
$$

which is a direct generalization of the first line of the right hand side of (53); as this term, it contains dynamical correlations at short distances.

Of course, as for $U_{2}$, there are terms in $\rho_{\mathrm{II}}$ that are second order in $U_{3}$ third order, etc. as well as terms depending of the higher order Ursell operators $U_{l}$. Nevertheless, for a gas, we will see that the terms that we have already explicitly written are sufficient for a good description of the correlations.

\section{Effects of a Strong Repulsion with Short Range}

We now study the properties of the partial density operator $\rho_{\mathrm{II}}$ for a potential with a strong repulsive core. For the sake of simplicity, we will often assume a hard sphere potential of spatial extension $b$ :

$$
V\left(\mathbf{r}_{1}, \mathbf{r}_{2}\right)= \begin{cases}\infty & \text { for } r=\left|\mathbf{r}_{1}-\mathbf{r}_{2}\right|<b \\ 0 & \text { otherwise }\end{cases}
$$

but most of our calculations are in fact more general, $b$ being defined as the distance between particles at which the interaction potential becomes significantly larger than the thermal energy $k_{\mathrm{B}} T$. We know that the two body density operator:

$$
\left\langle\mathbf{r}_{1}, \mathbf{r}_{2}\left|\rho_{\mathrm{II}}\right| \mathbf{r}_{1}^{\prime}, \mathbf{r}_{2}^{\prime}\right\rangle
$$

must necessarily vanish whenever ether the distance $\left|\mathbf{r}_{1}-\mathbf{r}_{2}\right|$, or $\left|\mathbf{r}_{1}^{\prime}-\mathbf{r}_{2}^{\prime}\right|$, (or both) becomes inferior to $b$. This is because, if we call $\left|\Psi_{k}\right\rangle$ the eigenstates of the $N$ particle Hamiltonian $H_{N}$

$$
H_{N}\left|\Psi_{k}\right\rangle=E_{k}\left|\Psi_{k}\right\rangle
$$


and $\Psi_{n}\left(\mathbf{r}_{1}, \mathbf{r}_{2}, \mathbf{r}_{3}, \ldots \mathbf{r}_{N}\right)$ the associated wave functions, the matrix elements of $\rho_{\mathrm{II}}^{\text {can }}$ can be expressed as:

$$
\begin{aligned}
\left\langle\mathbf{r}_{1}, \mathbf{r}_{2}\left|\rho_{\mathrm{II}}^{\text {can }}\right| \mathbf{r}_{1}^{\prime}, \mathbf{r}_{2}^{\prime}\right\rangle=\int \mathrm{d}^{3} r_{3} \ldots . . \int \mathrm{d}^{3} r_{N} \sum_{n} e^{-\beta E_{n}} \Psi_{n}\left(\mathbf{r}_{1}, \mathbf{r}_{2}, \mathbf{r}_{3}, \ldots \mathbf{r}_{N}\right) \times \\
\times \Psi_{n}^{*}\left(\mathbf{r}_{1}^{\prime}, \mathbf{r}_{2}^{\prime}, \mathbf{r}_{3}^{\prime}, \ldots \mathbf{r}_{N}^{\prime}\right)
\end{aligned}
$$

where the wave functions vanish exponentially in any region of configuration space where the potential energy exceeds their energy (in the limit of infinite potential and hard cores, they vanish exactly whenever two hard cores overlap). Therefore, if either $\left|\mathbf{r}_{1}-\mathbf{r}_{2}\right|$ or $\left|\mathbf{r}_{1}^{\prime}-\mathbf{r}_{2}^{\prime}\right|$ is less than $b$ (or both), the corresponding matrix elements of $\rho_{\mathrm{II}}^{\text {can }}$ have to vanish. It is straightforward to extend this result to the grand canonical version $\rho_{\mathrm{II}}$ of the two particle density operator.

Such a cancellation is a non-perturbative property of the two particle density operator. At zero relative distances, however small the potential range $b$ is, the correction brought by the interactions to the ideal gas expression of $\rho_{\text {II }}$ remains finite: it has to exactly compensate for the zero-order term. On the other hand, nothing similar occurs for the one particle density operator, which undergoes only perturbative changes. Mathematically speaking, hard core potentials introduce a non-analytical expression of the potential energy, and the limit of the matrix elements of $\rho_{\text {II }}$ at short distances is obtained in a non-unform convergence process. It is therefore an interesting test of our method to see how this result is recovered, and indeed we will see that the process is not trivial.

In what follows we limit ourselves to the proof of this non-perturbative cancellation: we look for all short range corrections in $\rho_{\text {II }}$ that are of zero-order in $b$ and ignore all higher order corrections. What we have to do, then, is to consider the whole series of terms of the Ursell expansion of $\rho_{\mathrm{II}}$ and identify the terms of zero-order in $b$; fortunately, it turns out that only a small numbers of the Ursell diagrams contribute, namely those of zero, first and second-order in $U_{2}$ as well as first-order in $U_{3}$. As in Section 3.2 above, we start the analysis from the terms which are zero-order in $U_{2}$.

4.1. ZERO-ORDER TERM IN $U_{2}$. - In a system with translational invariance (periodic boundary conditions), $\rho_{\text {II }}$ commutes with the sum of the momenta of the two particles, which means that all its matrix elements are diagonal with respect to this sum. We therefore have

$$
\begin{aligned}
\left\langle\mathrm{r}_{1}, \mathrm{r}_{2}\left|\rho_{\mathrm{II}}\right| \mathrm{r}_{1}^{\prime}, \mathbf{r}_{2}^{\prime}\right\rangle & =\mathcal{V}^{-2} \sum_{\mathbf{K}} \sum_{\mathbf{q}, \mathbf{q}^{\prime}} \mathrm{e}^{2\left[\mathbf{q}^{\prime} \cdot\left(\mathbf{r}_{1}^{\prime}-\mathbf{r}_{2}^{\prime}\right)-\mathbf{q}^{\prime} \cdot\left(\mathrm{r}_{1}-\mathbf{r}_{2}\right)\right]} \\
& \times\left\langle\frac{\mathbf{K}}{2}+\mathbf{q}, \frac{\mathbf{K}}{2}-\mathbf{q}\left|\rho_{\mathrm{II}}\right| \frac{\mathbf{K}}{2}+\mathbf{q}^{\prime}, \frac{\mathbf{K}}{2}-\mathbf{q}^{\prime}\right\rangle
\end{aligned}
$$

where $\mathcal{V}$ is the volume of the system (which arises from the normalization of the plane waves). This general formula can readily be applied to an ideal gas; from (29), where the operators $f$ are diagonal in the momentum representation, we obtain a direct term where $\mathbf{q}=\mathbf{q}^{\prime}$, followed by an exchange term where $\mathbf{q}=-\mathbf{q}^{\prime}$, so that the sum is

$$
\int \mathrm{d}^{3} k_{1} \int \mathrm{d}^{3} k_{2} f\left(\mathbf{k}_{1}\right) f\left(\mathbf{k}_{2}\right)\left[\mathrm{e}^{2\left(\mathbf{k}_{2}-\mathbf{k}_{1}\right) \cdot\left(\mathbf{r}_{1}-\mathbf{r}_{2}-\mathbf{r}_{1}^{\prime}+\mathbf{r}_{2}^{\prime}\right) / 2}+\eta \mathrm{e}^{2\left(\mathbf{k}_{2}-\mathbf{k}_{1}\right)\left(\mathbf{r}_{1}+\mathbf{r}_{1}^{\prime}-\mathbf{r}_{2}-\mathbf{r}_{2}^{\prime}\right) / 2}\right] .
$$

If for instance we consider only the diagonal elements of $\rho_{\mathrm{II}}\left(\mathbf{r}_{1}=\mathbf{r}_{1}^{\prime}, \mathbf{r}_{2}=\mathbf{r}_{2}^{\prime}\right)$, we see that the only spatial dependence arises from the exchange term (term in $\eta$ inside the bracket). More generally, in equation (79), the only characteristic length is the thermal wavelength introduced by the functions $f$

$$
\lambda_{\mathrm{T}}=\frac{h}{\sqrt{2 \pi m k_{\mathrm{B}} T}}
$$


and no special variation occurs when $\left|\mathbf{r}_{1}-\mathbf{r}_{2}\right|$ or $\left|\mathbf{r}_{1}^{\prime}-\mathbf{r}_{2}^{\prime}\right|$ becomes smaller than $b$. The result is, of course, independent of $b$, which makes it zero order in $b$ for any value of the r's. In the following calculation, therefore, our purpose will be to find in the Ursell expansion series the terms which will compensate for the short range value of (79) and to check that no other zero-order term has been omitted.

\subsection{FIRST-ORDER IN $U_{2}$}

4.2.1. Preliminary Considerations. - In order to simplify the discussion of the other terms, we first make a few remarks about the characteristic lengths involved in the matrix elements of some relevant operators. We start from the elements of $U_{2}$ itself

$$
\left\langle\mathbf{r}_{1}, \mathbf{r}_{2}\left|U_{2}(1,2)\right| \mathbf{r}_{1}^{\prime}, \mathbf{r}_{2}^{\prime}\right\rangle
$$

In this element, if the distance between two particles (in the bra- or in the -ket) is smaller than the potential range $b$, the application of the operator $\exp \left[-\beta\left(H_{0}(1,2)+V(1,2)\right)\right]$ gives zero; this is easily checked by a reasoning similar to that based on equation (76). In this case, inside (81), $U_{2}$ "reduces" according to

$$
U_{2}(1,2)=\mathrm{e}^{-\beta\left[H_{0}(1,2)+V(1,2)\right]}-\mathrm{e}^{-\beta H_{0}(1,2)} \Rightarrow-\mathrm{e}^{-\beta H_{0}(1,2)}=-U_{1}(1) U_{1}(2)
$$

Therefore, if at least one of the lengths $\left|r_{1}-r_{2}\right|$ and $\left|r_{1}^{\prime}-r_{2}^{\prime}\right|$ is smaller than $b$, that is "inside hard cores", we have

$$
\begin{aligned}
\left\langle\mathbf{r}_{1}, \mathbf{r}_{2}\left|U_{2}(1,2)\right| \mathbf{r}_{1}^{\prime}, \mathbf{r}_{2}^{\prime}\right\rangle= & -\left\langle\mathbf{r}_{1}, \mathbf{r}_{2}\left|U_{1}(1) U_{1}(2)\right| \mathbf{r}_{1}^{\prime}, \mathbf{r}_{2}^{\prime}\right\rangle \\
& =-\left[\lambda_{\mathrm{T}}\right]^{-6} \mathrm{e}^{-\left[\pi\left(\mathbf{r}_{1}-\mathbf{r}_{1}^{\prime}\right) / \lambda_{\mathrm{T}}\right]^{2}} \mathrm{e}^{-\left[\pi\left(\mathbf{r}_{2}-\mathbf{r}_{2}^{\prime}\right) / \lambda_{\mathrm{T}}\right]^{2}}
\end{aligned}
$$

Obviously, this matrix element is zero-order in $b$ (it is actually completely independent of the potential range); it has significant values as long as the differences $\left|r_{1}-r_{1}^{\prime}\right|$ and $\left|r_{2}-r_{2}^{\prime}\right|$ are not much larger than the thermal wavelength $\lambda_{\mathrm{T}}$.

For a more detailed study, since the motion of the center of mass factorizes inside $U_{2}$, it is convenient to introduce the variables $\mathbf{R}$ of the center of mass and $r$ of the relative motion and to write

$$
\left\langle\mathbf{R}, \mathbf{r}\left|U_{2}(1,2)\right| \mathbf{R}^{\prime}, \mathbf{r}^{\prime}\right\rangle=2^{3 / 2}\left[\lambda_{\mathrm{T}}\right]^{-3} e^{-\left[2 \pi\left(\mathbf{R}-\mathbf{R}^{\prime}\right) / \lambda_{\mathrm{T}}\right]^{2}}\left\langle\mathbf{r}\left|\mathbf{U}_{2}^{\text {rel }}\right| \mathbf{r}^{\prime}\right\rangle
$$

where $U_{2}^{\text {rel }}$ is given in terms of the operator $\mathbf{P}$ associated with the relative momentum by

$$
U_{2}^{\text {rel }}=e^{-\beta\left[\frac{\mathbf{P}^{2}}{1}+V_{12}(\mathbf{r})\right]}-e^{-\beta\left[\frac{\mathbf{P}^{2}}{r}\right]}
$$

We have

$$
\left\langle\mathbf{r}\left|U_{2}^{\text {rel }}\right| \mathbf{r}^{\prime}\right\rangle=\sum_{k} \mathrm{e}^{-\beta e_{k}} \varphi_{k}(\mathbf{r})\left[\varphi_{k}\left(\mathbf{r}^{\prime}\right)\right]^{*}-\sum_{k} \mathrm{e}^{-\beta e_{h}^{o}} \varphi_{k}^{o}(\mathbf{r})\left[\varphi_{k}^{o}\left(\mathbf{r}^{\prime}\right)\right]^{*}
$$

where the $e_{k}$ and the $\varphi_{k}(\mathbf{r})$ are the energies and the eigenfunctions of the Hamilonian $H_{\text {rel }}$ describing the relative motion in the presence of the interaction potential, while the same notations with an extra upper index zero are the corresponding quantities in the absence of interaction $\left({ }^{8}\right)$ Equation (84) shows that the $\mathbf{R}$ and $\mathbf{R}^{\prime}$ dependence are the same as for an ideal gas, explicitly given in (83); on the other hand, the $\mathbf{r}$ and $\mathbf{r}^{\prime}$ dependence is more complicated

$\left({ }^{8}\right)$ For simplicity and compactness of the notation we assume a finite volume and do not distinguish explicitly the discrete (bound) spectrum from the continuum. 
since, according to (86), it involves a difference of two exponential operators. We already know from the discussion above that, if $\mathrm{r}$ or $\mathrm{r}^{\prime}$ (or both), are smaller than $b, U_{2}^{\text {rel }}$ merely reduces to the second term of (86), associated with a free relative motion, so that the matrix element becomes zero-order in $b$. Let us assume then that $\mathbf{r}^{\prime}$ is fixed to some value, for instance of the order of $\lambda_{\mathrm{T}}$, and that $\mathbf{r}$ increases progressively from zero. In three dimensions, the perturbation of the eigen-wave functions of the Hamiltonian, introduced by a short range potential, is limited within a region $\left({ }^{9}\right)$ of size comparable to the range. As a consequence, we expect that the matrix element of $U_{2}^{\text {rel }}$ will remain of zero-order in $b$ only within a domain of dimension comparable to $b$ (or some relatively small multiple of this length). If, on the other hand, $\mathbf{r}$ increases beyond this domain, the matrix elements will decrease (in absolute value) and become first-order in $b$, for instance proportional to $b / \lambda_{\mathrm{T}}$. In other words, sufficiently far from the origin they tend uniformly towards zero when $b$ tends to zero, as opposed to what happens when $\mathbf{r}$ is comparable to $b$.

Since the two variables $\mathbf{r}$ and $\mathbf{r}^{\prime}$ play a symmetric role in the discussion, we may summarize this discussion by saying that the matrix elements of $U_{2}$ are first-order in $b$ everywhere except in regions of the configuration space where either $|\mathbf{r}|$, or $\left|\mathbf{r}^{\prime}\right|$, or both, are of the order of the potential range $b$.

We now assume that, inside (81), we insert an operator $f$, for instance on the right hand side of $U_{2}$, so that we study the matrix element

$$
\left\langle\mathbf{r}_{1}, \mathbf{r}_{2}\left|U_{2}(1,2) f(1)\right| \mathbf{r}_{1}^{\prime}, \mathbf{r}_{2}^{\prime}\right\rangle=\int \mathrm{d}^{3} r\left\langle\mathbf{r}_{1}, \mathbf{r}_{2}\left|U_{2}(1,2)\right| \mathbf{r}_{1}^{\prime}+\mathbf{r}, \mathbf{r}_{2}^{\prime}\right\rangle\left\langle\mathbf{r}_{1}^{\prime}+\mathbf{r}|f| \mathbf{r}_{1}^{\prime}\right\rangle
$$

We first note that, if $\left|\mathbf{r}_{1}-\mathbf{r}_{2}\right| \leq b$, this matrix element is zero-order in $b$ since $U_{2}$ reduces to a product of $U_{1}$ 's as in (82); the dependence in $\left|\mathbf{r}_{1}-\mathbf{r}_{2}\right|$ is therefore of the same type as that obtained in the absence of an operator $f$. But if $\left|\mathbf{r}_{1}-\mathbf{r}_{2}\right|$ has much larger values, for instance of the order of $\lambda_{\mathrm{T}}$, the dependence in $\left|\mathbf{r}_{1}^{\prime}-\mathbf{r}_{2}^{\prime}\right|$ is drastically affected by the intermediate operator $f$ : however small $\left|\mathbf{r}_{1}^{\prime}-\mathbf{r}_{2}^{\prime}\right|$, the matrix elements never come back to zero-order in $b$. To show this, we remark that, as for $U_{1}$, the only characteristic length which occurs in $f$ is the thermal wavelength $\lambda_{\mathrm{T}}$, which is necessarily much larger than $b$ if the latter length tends to zero. In other words, in the integration over $\mathrm{d}^{3} r$ of (87), the second matrix element has significant values in a range centered around the origin and of size comparable to $\lambda_{\mathrm{T}}$; one may see this as the result of a "delocalization" of particle (1) in the ket produced by the operator $f$. As for the first matrix element, it has two characteristic lenghts, $b$ and $\lambda_{\mathrm{T}}$, so that we must distinguish in the integration domain two contributions: first, that arising from a volume comparable to $b^{3}$ around the value $\mathbf{r}=\mathbf{r}_{1}^{\prime}-\mathbf{r}_{2}^{\prime}$, which obviously gives a contribution of third order $\left({ }^{10}\right)$ in $b$ which we can Ignore here; second, that arising from the rest of the volume where the function to be integrated itself is first-order in $b$. The net result is that, provided $\left|\mathbf{r}_{1}-\mathbf{r}_{2}\right|$ sufficently large, however small $\left|\mathbf{r}_{1}^{\prime}-\mathbf{r}_{2}^{\prime}\right|$ is, the matrix element (87) always remains of first-order in $b$ and tends uniformly to zero when the potential range decreases. To make it zero-order in $b$ necessitates that the distance of the particles in the bra which touches directly $U_{2}$ is allowed to become very small. By symmetry, it is straightforward to obtain similar results

$\left({ }^{9}\right)$ This property arises from the fact that the three dimension problem is equivalent to a one dimension problem with a boundary condition which always cancels the fictitious wave function at the orıgin, even in the absence of a potential; this phenomenon does not take place for lower dimension problems.

$\left({ }^{10}\right)$ The volume where the matrix element of $U_{2}$ is first-order in $b$ does not have to be precisely proportional to $b^{3}$ for our reasoning to be valid; it could vary more slowly, as $b\left[\lambda_{T}\right]^{2}$ for instance, without affecting our results. 
for an operator $f$ acting on the left of an operator $U_{2}$; it is sufficient to interchange the bras and the kets in the reasoning.

Finally, if we consider the matrix element

$$
\left\langle\mathbf{r}_{1}, \mathbf{r}_{2}\left|f(1) U_{2}(1,2) f(1)\right| \mathbf{r}_{1}^{\prime}, \mathbf{r}_{2}^{\prime}\right\rangle
$$

or

$$
\left\langle\mathbf{r}_{1}, \mathbf{r}_{2}\left|f(2) U_{2}(1,2) f(1)\right| \mathbf{r}_{1}^{\prime}, \mathbf{r}_{2}^{\prime}\right\rangle
$$

A simple generalization of our reasoning shows that, since the bras and kets where the positions of the particles are fixed are never in direct contact with $U_{2}$ (but only through $f$ operators that delocalize them over about $\lambda_{\mathrm{T}}$ ), the matrix elements are always at least of first-order in $b$; they tend uniformly towards zero with $b$ everywhere. Moreover, all preceding results apply without any change when $U_{2}$ is replaced by $U_{2}^{\mathrm{S}, \mathrm{A}}$ which, according to (48), contains an extra operator $P_{\text {ex. }}$, this is because the exchange operator changes the sign of the vector $\mathbf{r}_{1}-\mathbf{r}_{2}$ but not its length. To summarize, zero-order terms in $b$ can only be obtained when the $U_{2}^{\mathrm{S} \text {, } \mathrm{A}}$ operator is in direct contact with the ket, or the bra, where two particles are at a short distance (of the order of $b$ ).

4.2.2. Contribution to $\rho_{\mathrm{II}}$. - We now apply the preceding results to equations (51)-(53). Two types of corrections are present in $\rho_{\mathrm{II}}$, those which appear through changes of $\rho_{\mathrm{I}}$ and contain no dynamical correlations, and those which are given by (53) which do contain such correlations. It is natural to expect that the former do not play a role in the present discussion, and this is indeed the case because they contain a trace over a collision partner which makes them at least first-order in $b$. Mathematically we have

$$
\left\langle\mathbf{r}_{1}\left|\operatorname{Tr}_{2}\left\{U_{2}^{\mathrm{S}, \mathrm{A}}[1+\eta f(2)]\right\}\right| \mathbf{r}_{2}^{\prime}\right\rangle=\int \mathrm{d}^{3} r_{2}\left\langle\mathbf{r}_{1}, \mathbf{r}_{2}\left|U_{2}^{\mathrm{S}, \mathrm{A}}[1+\eta f(2)]\right| \mathbf{r}_{1}^{\prime}, \mathbf{r}_{2}\right\rangle
$$

Since an integration over a variable $\mathrm{d}^{3} r_{2}$ is involved, the situation is similar to that found in (87): one may distinguish two domains of integration, one where the matrix elements are zero-order but which has a volume tending to zero with $b$, and one where the matrix elements themselves tend to zero, so that the sum is at least first-order in $b$. So, we only have to include (53) in our zero-order reasoning. We now distinguish between three cases:

i) If $\left|\mathbf{r}_{1}^{\prime}-\mathbf{r}_{2}^{\prime}\right| \leq b$ while $\left|\mathbf{r}_{1}-\mathbf{r}_{2}\right|$ is much larger, according to what has been said in Section 4.2.1, the zero-order terms inside (53) are obtained by suppression of the $f$ operators from the brackets $[1+\eta f]$ 's on the right of $U_{2}^{\mathrm{S}, \mathrm{A}}$. This is because the operators $f$ 's would introduce, according to (87), corrections that tend uniformly to zero when $b$ tends to zero. Under these conditions the matrix elements we obtain are those of

$$
2 z^{2}\left\langle\mathbf{r}_{1}, \mathbf{r}_{2}\left|[1+\eta f(1)][1+\eta f(2)] U_{2}^{\mathrm{S}, \mathrm{A}}(1,2)\right| \mathbf{r}_{1}^{\prime}, \mathbf{r}_{2}^{\prime}\right\rangle
$$

but, since $U_{2}$ reduces to a product of $U_{1}$ 's according to (82), we merely obtain the result

$$
-\left\langle\mathbf{r}_{1}, \mathbf{r}_{2}\left|f(1) f(2)\left[1+\eta P_{\text {ex }}\right]\right| \mathbf{r}_{1}^{\prime}, \mathbf{r}_{2}^{\prime}\right\rangle
$$

which shows that the ideal gas term (29) is exactly cancelled by this term; we therefore obtain the expected cancellation of the matrix element of $\rho_{\text {II }}$ in this case.

ii) If $\left|\mathbf{r}_{1}^{\prime}-\mathbf{r}_{2}^{\prime}\right| \leq b$ while $\left|\mathbf{r}_{1}-\mathbf{r}_{2}\right|$ is much larger, the reasoning is exactly similar to that of (i), except that one has to interchange bras and kets. In this case also, the first-order terms in $U_{2}$ are exactly opposed to the zero-order terms and the matrix elements of $\rho_{\mathrm{II}}$ vanıs as required. 
iii) If $\left|\mathbf{r}_{1}-\mathbf{r}_{2}\right|$ and $\left|\mathbf{r}_{1}^{\prime}-\mathbf{r}_{2}^{\prime}\right|$ are both smaller than $b$, in order to provide zero-order contributions in $b$, the reduction process of $U_{2}^{\mathrm{S}, \mathrm{A}}$ may occur, either from the bra or from the ket (or both). We may therefore keep the factors $[1+\eta f(1)][1+\eta f(2)]$ either on the right, or on the left of the operator $U_{2}^{\mathrm{S}, \mathrm{A}}$, but in doing so we double count the term where all brackets $[1+\eta f]$ are replaced by one, so that it is necessary to remove this contribution. The total result is therefore

$$
\begin{array}{r|r|r}
2 z^{2}\left\langle\mathbf{r}_{1}, \mathbf{r}_{2}\right. & {[1+\eta f(1)][1+\eta f(2)] U_{2}^{\mathrm{S}, \mathrm{A}}(1,2)} & \left.\mathbf{r}_{1}^{\prime}, \mathbf{r}_{2}^{\prime}\right\rangle+ \\
+2 z^{2}\left\langle\mathbf{r}_{1}, \mathbf{r}_{2}\right| & U_{2}^{\mathrm{S}, \mathrm{A}}(1,2)[1+\eta f(1)][1+\eta f(2)] & \left.\mathbf{r}_{1}^{\prime}, \mathbf{r}_{2}^{\prime}\right\rangle- \\
-2 z^{2}\left\langle\mathbf{r}_{1}, \mathbf{r}_{2}\right| U_{2}^{\mathrm{S}, \mathrm{A}}(1,2) & \left.\mathbf{r}_{1}^{\prime}, \mathbf{r}_{2}^{\prime}\right\rangle
\end{array}
$$

which is equal to

$$
-2\left\langle\mathbf{r}_{1}, \mathbf{r}_{2}\left|f(1) f(2)\left[1+\eta P_{\mathrm{ex}}\right]\right| \mathbf{r}_{1}^{\prime}, \mathbf{r}_{2}^{\prime}\right\rangle+z^{2}\left\langle\mathbf{r}_{1}, \mathbf{r}_{2}\left|U_{1}(1) U_{1}(2)\left[1+\eta P_{\mathrm{ex}}\right]\right| \mathbf{r}_{1}^{\prime}, \mathbf{r}_{2}^{\prime}\right\rangle
$$

Therefore, in the case where the hard cores of the particles overlap in both the bra and the ket, the zero-order cancellation with (29) is no longer obtained. We will see that higher order terms are needed to restore it.

4.3. NEXT Terms. - We have seen in the preceding section that taking into account only diagrams with at most one $U_{2}$ is not sufficient for describing the short range properties of $\rho_{\text {II }}$ correctly. We will now search for other terms, derived from other diagrams, which give a contribution of order $b^{0}$ to $\rho_{\mathrm{II}}$, in order to see whether, by this way, we can obtain a faithful description of what happens at short interparticle distances.

4.3.1. Second-Order in $U_{2}$. - We now consider the contribution of the diagrams with two $U_{2}$ 's given in Section 3.3. The first term we study is given by (63). We are searching for a contribution of zero-order in the potential range, which can only be done by reducing each of the two $U_{2}$ 's into two $U_{1}$ 's; this means that both $U_{2}$ 's must act directly on bras or kets where two particles are close (we have seen in the preceding section that every $f$ operating on a two particle wave function delocalizes one of the particles, which introduces a supplementary order in $b$ ). Hence, from (63), we keep only the contribution

$$
2 z^{4} U_{2}^{\mathrm{S}, \mathrm{A}}(1,2)[1+\eta f(1)][1+\eta f(2)] U_{2}^{\mathrm{S}, \mathrm{A}}(1,2) .
$$

Therefore, if $\left|\mathbf{r}_{1}-\mathbf{r}_{2}\right|$ and $\left|\mathbf{r}_{1}^{\prime}-\mathbf{r}_{2}^{\prime}\right|$ are both smaller than $b$, we have to add to (94) the following contribution

$$
z^{2}\left\langle\mathbf{r}_{1} \mathbf{r}_{2}\left|f(1) f(2) U_{1}(1) U_{1}(2)\left[1+\eta P_{\mathrm{ex}}(1,2)\right]\right| \mathbf{r}_{1}^{\prime} \mathbf{r}_{2}^{\prime}\right\rangle
$$

On the other hand, if $\left|\mathbf{r}_{1}^{\prime}-\mathbf{r}_{2}^{\prime}\right| \leq b$ while $\left|\mathbf{r}_{1}-\mathbf{r}_{2}\right|$, or the reverse, we have no extra term to add to (92).

As for the term given in (60), it involves a trace over a third particle and can be ignored. This is because one can show - following the same line as in the previous section - that the trace introduces a supplementary order in $b$. The reasoning can be generalized: any trace over a third particle gives a contribution which is of at least order $b$, as long as there is no permutation operator inside the trace which transposes the two particles $\rho_{\text {II }}$ is operating on (see the discussion of the terms with one $U_{3}$ later in this section). Hence, we will be able to drop all terms with a trace, so that the only contribution to $\rho_{\text {II }}$ of the diagrams with two $U_{2}$ in the order considered arises when the particles both in the bra and the ket are closer than $b$, and is then given by the expression derived above (96). 
4.3.2. First-Order in $U_{3}$. - The next terms of the Ursell series expansion of $\rho_{\text {II }}$ are the contribution to $\log Z$ coming from the diagrams containing one single $U_{3}$, which we have obtained in Section 3.4. Since both (71) and (72) involve a trace over (at least) one extra particle, at first sight, one might infer from the discussion of the previous section that they introduce only matrix elements that tend uniformly towards zero with $b$. We will see that this is not true, and that some new zero-order contribution is introduced by the exchange terms.

We start with the second term (72) which contains only one trace, so that it is more likely to leave a zero-order contribution. As in the previous section, we can ignore the $f$ 's acting at both ends on particle 1 or 2 , since they correspond to terms which tend to zero with $b$, so that the matrix element becomes

$$
\left.6 z^{3}<\mathbf{r}_{1}, \mathbf{r}_{2}\left|\operatorname{Tr}_{3}\left\{U_{3}^{\mathrm{S}, \mathrm{A}}(1,2,3)[1+\eta f(3)]\right\}\right| \mathbf{r}_{1}^{\prime}, \mathbf{r}_{2}^{\prime}\right\rangle .
$$

How can $U_{3}$ be reduced in this term to give a zero-order contribution? The operator $U_{3}$ is defined as

$$
U_{3}=\mathrm{e}^{-\beta H(1,2,3)}-\left[U_{1}(1) U_{2}(2,3)+\text { permutations }\right]-U_{1}(1) U_{1}(2) U_{1}(3) .
$$

If two particles, for instance 1 and 2 , are at a distance smaller than $b$ inside either a bra or a ket on which $U_{3}$ acts, the cancellation of the three body wave functions inside hard cores allows us to ignore the term in $\mathrm{e}^{-\beta H(1,2,3)}$ and to reduce one of the $U_{2}$ 's so that $U_{3}$ becomes

$$
U_{3} \rightarrow-U_{1}(1) U_{2}(2,3)-U_{1}(2) U_{2}(1,3)
$$

If, in addition, we assume that in either the bra or the ket the distance between particle 3 and particle 2 is smaller than $b$, the operator $U_{3}$ is further reduced according to

$$
U_{3} \rightarrow U_{1}(1) U_{1}(2) U_{1}(3)-U_{1}(2) U_{2}(1,3) .
$$

We note that the first term is indeed of order zero in $b$, whereas we cannot make any general statement about the second term (as long as we do not specify anything about the distance between the first and the third particles).

We can now return to (97) and develop the symmetrizer/antisymmetrizer according to

$$
\frac{1}{6}\left\{1+\eta\left[P_{\mathrm{ex}}(1,2)+P_{\mathrm{ex}}(1,3)+P_{\mathrm{ex}}(2,3)\right]+C_{3}(1,2,3)+C_{3}(1,3,2)\right\}
$$

and examine the terms one by one, assuming that particles 1 and 2 are close (in the bra).

i) The direct term

$$
\left.z^{3}<\mathbf{r}_{1}, \mathbf{r}_{2}\left|\operatorname{Tr}_{3}\left\{U_{3}(1,2,3)[1+\eta f(3)]\right\}\right| \mathbf{r}_{1}^{\prime}, \mathbf{r}_{2}^{\prime}\right\rangle
$$

can be reduced once to

$$
-z^{3}<\mathbf{r}_{1}, \mathbf{r}_{2}\left|\operatorname{Tr}_{3}\left\{\left[U_{1}(1) U_{2}(2,3)+U_{1}(2) U_{2}(1,3)\right][1+\eta f(3)]\right\}\right| \mathbf{r}_{1}^{\prime}, \mathbf{r}_{2}^{\prime}>
$$

but not further; the trace over the third particle introduces an order $b$ by the same process as in the discussion of $U_{2}$-terms. The contribution of the direct term therefore vanishes to zero-order in $b$, whatever the positions of particles 1 and 2 are.

ii) The term where particles 1 and 2 are exchanged

$$
\eta z^{3}<\mathbf{r}_{1}, \mathbf{r}_{2}\left|\operatorname{Tr}_{3}\left\{U_{3}(1,2,3) P_{\text {ex }}(1,2)[1+\eta f(3)]\right\}\right| \mathbf{r}_{1}^{\prime}, \mathbf{r}_{2}^{\prime}>
$$


which, after a first reduction, becomes

$$
-\eta z^{3}<\mathbf{r}_{1}, \mathbf{r}_{2}\left|\operatorname{Tr}_{3}\left\{\left[U_{1}(1) U_{2}(2,3)+U_{1}(2) U_{2}(1,3)\right] P_{\mathrm{ex}}(1,2)[1+\eta f(3)]\right\}\right| \mathbf{r}_{1}^{\prime}, \mathbf{r}_{2}^{\prime}>
$$

Here again we cannot reduce $U_{3}$ anymore, since both $U_{2}$ 's act on the third particle over which the trace is taken. For the same reasons as before, this leads to a negligible contribution.

iil The terms where the third particle is exchanged with particle 1 or 2 ; the former term is given by

$$
\eta z^{3}<\mathbf{r}_{1}, \mathbf{r}_{2}\left|\operatorname{Tr}_{3}\left\{U_{3}(1,2,3) P_{\mathrm{ex}}(1,3)[1+\eta f(3)]\right\}\right| \mathbf{r}_{1}^{\prime}, \mathbf{r}_{2}^{\prime}>
$$

Assuming that particle 1 and 2 are close in the bra, we apply (99) and obtain, by inserting a closure relation and making the trace over the third particle explicit

$$
\begin{array}{r}
-\eta z^{3} \int \mathrm{d}^{3} r_{3} \mathrm{~d}^{3} r<\mathbf{r}_{1}, \mathbf{r}_{2}, \mathbf{r}_{3}\left|\left[U_{1}(1) U_{2}(2,3)+U_{1}(2) U_{2}(1,3)\right]\right| \mathbf{r}, \mathbf{r}_{2}^{\prime}, \mathbf{r}_{1}^{\prime}>\times \\
\times<\mathbf{r}|[1+\eta f]| \mathbf{r}_{3}>
\end{array}
$$

The term in $U_{1}(1) U_{2}(1,3)$ does not introduce any zero-order contribution since there is no way to reduce $U_{2}(1,3)$, whatever values we choose for $\mathbf{r}_{1}^{\prime}$ and $\mathbf{r}_{2}^{\prime}$; we therefore ignore it. However, the term in $U_{1}(1) U_{2}(2,3)$ can indeed be reduced from the right hand side by assuming that $\left|\mathbf{r}_{1}^{\prime}-\mathbf{r}_{2}^{\prime}\right| \leq b$, leading to the result

$$
\eta z^{3}<\mathbf{r}_{1}\left|U_{1}[1+\eta f] U_{1}\right| \mathbf{r}_{1}^{\prime}><\mathbf{r}_{2}\left|U_{1}\right| \mathbf{r}_{2}^{\prime}>=\eta z^{2}<\mathbf{r}_{1}, \mathbf{r}_{2}\left|U_{1}(1) U_{1}(2) f(1)\right| \mathbf{r}_{1}^{\prime}, \mathbf{r}_{2}^{\prime}>
$$

which is of the required zero-order.

Obviously the term in $P_{\text {ex }}(2,3)$ of $(101)$ leads to a simllar contribution, where the indices 1 and 2 are exchanged. We therefore have to add the following zero-order contribution to $\rho_{\mathrm{II}}$ :

$$
\eta z^{2} U_{1}(1) U_{1}(2)[f(1)+f(2)]
$$

1v) The cyclic permutation terms, of which the first one is given by

$$
z^{3}<\mathbf{r}_{1}, \mathbf{r}_{2}\left|\operatorname{Tr}_{3}\left\{U_{3}(1,2,3) C_{3}(1,2,3)[1+\eta f(3)]\right\}\right| \mathbf{r}_{1}^{\prime}, \mathbf{r}_{2}^{\prime}>
$$

which, according to (99), becomes, if $\left|\mathbf{r}_{1}-\mathbf{r}_{2}\right| \leq b$,

$$
\begin{array}{r}
-z^{3} \int \mathrm{d}^{3} r_{3} \mathrm{~d}^{3} r<\mathbf{r}_{1}, \mathbf{r}_{2}, \mathbf{r}_{3}\left|\left[U_{1}(1) U_{2}(2,3)+U_{1}(2) U_{2}(1,3)\right]\right| \mathbf{r}_{2}^{\prime}, \mathbf{r}, \mathbf{r}_{1}^{\prime}>\times \\
\times<\mathbf{r}|[1+\eta f]| \mathbf{r}_{3}>
\end{array}
$$

If, in addition, $\left|\mathbf{r}_{1}^{\prime}-\mathbf{r}_{2}^{\prime}\right| \leq b$, by the same argument as in (iii) we keep only the term in $U_{2}(1,3)$ which reduces into $U_{1}(1) U_{1}(3)$. This provides the result

$$
z^{2}<\mathbf{r}_{1}, \mathbf{r}_{2}\left|U_{1}(1) U_{1}(2) f(2) P_{\mathrm{ex}}(1,2)\right| \mathbf{r}_{1}^{\prime}, \mathbf{r}_{2}^{\prime}>
$$

Adding the equivalent contribution from the term in $C_{3}(1,3,2)$, we obtain the contribution to $\rho_{\text {II }}$ as:

$$
z^{2} U_{1}(1) U_{1}(2)[f(1)+f(2)] P_{e x}(1,2)
$$

The sum of all corrections to $\rho_{\text {II }}$ that we have obtained in this section is

$$
z^{2} U_{1}(1) U_{1}(2)[f(1)+f(2)]\left[\eta+P_{e x}(1,2)\right]
$$


which is valid whenever $\left|\mathbf{r}_{1}-\mathbf{r}_{2}\right| \leq b$ and $\left|\mathbf{r}_{1}^{\prime}-\mathbf{r}_{2}^{\prime}\right| \leq b$. On the other hand, if any of the distances $\left|\mathbf{r}_{1}-\mathbf{r}_{2}\right|$ or $\left|\mathbf{r}_{1}^{\prime}-\mathbf{r}_{2}\right|$ is much larger than $b$ (or both), there is no way to reduce all $U_{2}$ 's and no new zero-order term appears.

The other contribution to $\rho_{\mathrm{II}}$ originating from first-order in $U_{3}$ is given by (71) and contains the trace over two extra particles. While it was possible to cancel the effect of one extra trace in (72) by taking into account exchange operators, one can easily convince oneself that two traces are too many and that at least one trace over a $U_{2}$ operator will survive. All these terms tend to zero with $b$ and we may ignore them.

We conclude that the only term arising from diagrams with one $U_{3}$ which survives the reduction of $\rho_{\mathrm{II}}$ to zero-order zero in $b$ is (114); it contributes to $\rho_{\mathrm{II}}$ only when the interparticle distances in both the bra and the ket are smaller than $b$.

4.3.3. Higher-Order Terms. - We have not studied terms which are more than second-order in $U_{2}$ or first order in $U_{3}$. However, this is not necessary as long as we limit the calculations to zero-order in $b$. For instance, any contribution from diagrams with more than two $U_{2}$ 's will contain more second-order operators than it is possible to reduce by choosing close positions for two particles inside the bra and the ket; this is for example the case for the higher-order contributions resummed by ladder diagrams (cf. Appendix II). The same conclusion applies to second, third, etc. order in $U_{3}$ : we have seen that it is necessary to reduce $U_{3}$ twice, that is once from each side, in order to obtain a term which involves $U_{1}$ 's only, and completely reducing two independent $U_{3}$ 's is not possible. Generalizing to higher order Ursell operators, we may notice that in each term of the Ursell series expansion, we have to reduce all $U_{l}$ 's to $l U_{1}$ operators, in order to obtain contributions of zero-order in $b$. As we have at maximum two correlated particle pairs (in the bra and the ket) for reduction, it is clear that higher order terms $\left(U_{4}, 2 \times U_{3}\right.$, etc.) cannot be reduced completely to $U_{1}$ 's.

Therefore, the terms that we have calculated so far are sufficient for the study of the zeroorder properties of $\rho_{\mathrm{II}}$. Of course, if we were interested in higher order correlations between the particles, we would have to consider higher order partial density operators $\rho_{\text {III }}$, $\rho_{\text {IV }}$, etc. and the situation would be different: with more position variables in the bra and the ket, it would be possible to reduce more efficiently $U_{l}$ operators than inside $\rho_{\mathrm{II}}$, and to block more trace operations. Consequently, more complex diagrams would play a role to zero-order in $b$ when the distance between all particles in the bra and the ket is smaller than the potential range.

4.4. Summary. - To summarize, if either $\left|\mathbf{r}_{1}-\mathbf{r}_{2}\right|$ or $\left|\mathbf{r}_{1}^{\prime}-\mathbf{r}_{2}^{\prime}\right|$ is much smaller than $b$ (but not both), the first-order $U_{2}$ calculation which provides (53) is sufficient since it ensures a proper cancellation of $\rho_{\mathrm{II}}$ when we add it to the ideal gas term (29). On the other hand, if both $\left|\mathbf{r}_{1}-\mathbf{r}_{2}\right|$ and $\left|\mathbf{r}_{1}^{\prime}-\mathbf{r}_{2}^{\prime}\right|$ are smaller than $b$, the situation is more complex and we have to add the corrections given in equations (94), (96) and (114). The overall result is:

$$
\left\{-2 f(1) f(2)+z^{2} U_{1}(1) U_{1}(2)[1+f(1) f(2)+\eta f(1)+\eta f(2)]\right\} \times\left[1+\eta P_{\text {ex. }}\right]
$$

which, when added to (29), gives exactly zero, as readily seen from the relation

$$
f=z U_{1}[1+\eta f] \text {. }
$$

This shows that the matrix elements of $\rho_{\text {II }}$ vanish exactly inside hard cores, as expected, and that the sum of the three corrections gives a correct treatment of non-perturbative effects inside the potential; one can therefore expect that this expression provides a good description of the 
short range properties of $\rho_{\mathrm{II}}$. It is interesting that the cancellation of the matrix elements requires adding to the first and second order $U_{2}$ terms a contribution arising from a rather subtle exchange effect inside the $U_{3}$ term, corresponding to cyclic exchange or to exchange with an interaction partner.

To summarize, a good approximation to the two-body density operator $\rho_{\mathrm{II}}$, which includes perturbative effects at long relative distances between the particles as well as non-perturbative effects at short distances, is given by the following expression

$$
\begin{aligned}
\rho_{\mathrm{II}}=[ & f(1)+\delta f(1)] \otimes[f(2)+\delta f(2)]\left[1+\eta P_{\mathrm{ex}}\right]+ \\
& +[1+f(1)][1+f(2)] F(1,2)[1+f(1)][1+f(2)]
\end{aligned}
$$

where $\delta f$ is the operator defined in (52) and where

$$
F=2 z^{2} U_{2}^{\mathrm{S}, \mathrm{A}}+2 z^{4} U_{2}^{\mathrm{S}, \mathrm{A}}[1+f(1)][1+f(2)] U_{2}^{\mathrm{S}, \mathrm{A}}+6 z^{3} \operatorname{Tr}\left\{U_{3}^{\mathrm{S}, \mathrm{A}}(1,2,3)[1+f(3)]\right\}
$$

\section{Conclusion}

The U-C diagram method provides explicit expressions for the partial density operators, including non-perturbative effects. This is seen in equations (51), or the more precise approximation (117), which are the main results of the present article. The expressions remain relatively compact, at least when the calculation is limited to first-order in the Ursell operators. The two-body density operator gives physical information on the correlations between particles; the operator can be used to calculate, for instance, the amount of light scattered by a gaseous sample, or of density corrections to the index of refraction of a degenerate gas of bosons [7]. But other calculations are also possible. For instance, from the one-body density operator one can calculate average kinetic energy of the particles, from the two-body density operator the average potential energy, so that it is possible to get information on how the total energy is shared between its two components. Of course, for an exact calculation including all Ursell operators to all orders, the sum of the two energies should reconstruct exactly the "macroscopic" energy obtained by derivation of $\log Z$ with respect to the inverse temperature. But, for approximate calculations, this is not necessarly the case, so that the two calculations of energy give a convenient way to evaluate the quality of the approximations made in the calculations. Another check is given by the calculation of the pressure from the expression of its exact microscopic operator [8]; ideally, the average value of this operator over $\rho_{\text {II }}$ should reconstruct the macroscopic value $[k T \log Z] / \mathcal{V}$, so that deviations from this rule are a convenient guide in the approximations. Finally, the knowledge of $\rho_{\text {II }}$ could be useful as a tool for understanding the physical origin of complex phenomena such as the superfluid transition in gases, a subject that we are planning to investigate in future work.

It is also possible to take a point of view which is completely different from that in the present article, where the Ursell operators are known differences between exponentials of Hamiltonians, and to consider them as unknown operators which will be determined according to variational principles. The $N$ body density operator at thermal equilibrium is given by:

$$
\rho_{N}=\frac{1}{Z_{N}} K_{N}
$$

where $K_{N}$ is defined by the exact expression(3). Now, this expression can also be truncated to the terms leading to some approximation for $\log Z$ (first-order approximation terms in $U_{2}$ for instance, or second-order in $U_{2}$ with first-order $U_{3}$, etc.) while the $U_{l}$ are now left free to vary, instead of being fixed. This provides a variational $\rho_{N}$ or, by a simple generalization, a 
variational expression of the many particle density operator in the grand canonical ensemble. Now, since the expressions of the partial density operators that we have written allow an exact evaluation of the energy (or of the pressure, i.e. the grand potential), one can then determine the $U_{l}$ 's that make these expressions stationary. In this way one gets a variational method, valid at zero temperature, which provides approximate values of the ground state energy of the system. Of course, if no truncation had been in (3), the method should simply lead to the initial definition of the $U_{l}$ 's (at zero temperature) and to the exact energy. On the other hand, a truncation will in general lead to different expressions of the $U_{l}$ 's; for instance, if the operators of order more than 2 are ignored, the changes in the value of $U_{2}$ will tend to make up for the omission of $U_{3}$, etc. An interesting feature of the method is that is would automatically provide the next step in the variational method: inserting more U-C diagrams in the expression of $\log Z$. The extension to non-zero temperature can also be envisaged, by calculating the microscopic expression of the pressure and making it stationary, but the practical usefulness of this possibility has not yet been examined.

\section{Acknowledgments}

The authors are very grateful to Roger Balian for useful and stimulating discussions that were of great help to them.

\section{Appendix A}

Instead of varying the first Ursell operator $U_{1}$, as in the text, we can also obtain reduced density operators by varying the interaction potential. Let us assume that $V_{\imath \jmath}$ gets the following variation

$$
V_{\imath \jmath} \Rightarrow V_{\imath \jmath}+\beta^{-1} \mathrm{~d} x\left|i: \varphi_{a} ; j: \varphi_{b}\right\rangle\left\langle i: \theta_{a} ; j: \theta_{b}\right|
$$

Circular permutations under the trace allow us to write

$$
\begin{aligned}
& \mathrm{d} Z=\mathrm{d} x \operatorname{Tr}_{1,2, . . N}\left\{\sum_{i<3}\left|i: \varphi_{a} ; j: \varphi_{b}\right\rangle\left\langle i: \theta_{a} ; j: \theta_{b}\right| \mathrm{e}^{-\beta H_{N}} S\right\} \\
& =\frac{1}{2} Z \times\left\langle\theta_{a}, \theta_{b}\left|\rho_{\mathrm{II}}\right| \varphi_{a}, \varphi_{b}\right\rangle
\end{aligned}
$$

where $H_{N}$ is the $N$ particle Hamiltonian; the factor $1 / 2$ arises from the fact that $\rho_{\text {II }}$ is normalized with a trace equal to $N(N-1)$, and not to $N(N-1) / 2$. We therefore have the simple relation

$$
\left\langle\theta_{a}, \theta_{b}\left|\rho_{\mathrm{II}}\right| \varphi_{a}, \varphi_{b}\right\rangle=\left.2 \frac{\mathrm{d}}{\mathrm{d} x}\right|_{x=0} \log Z .
$$

If we apply this formula to an exact expression of $\log Z$, containing all terms of the expansion in Ursell operators, we will get exactly the same result as when using (18). But, if we start from some approximate expressions of $\log Z$, different results are in general obtained by the two methods. As an illustration, we apply (A.3) to the lowest order treatment of the interactions given by (33); because $U_{1}$ does not depend on the interaction potential the only variation of Log $Z$ arises from that of $U_{2}$, which is given by

$$
\begin{aligned}
\mathrm{d} U_{2}^{\mathrm{S}, \mathrm{A}}=\mathrm{d} x \sum_{n=0}^{\infty} \sum_{p=0}^{n-1}\left\{\frac{1}{n !}\left[-\beta H_{2}(1,2)\right]^{p}\right. & \left|1: \varphi_{a} ; 2: \varphi_{b}\right\rangle\left\langle 1: \theta_{a} ; 2: \theta_{b}\right| \times \\
& \left.\times\left[-\beta H_{2}(1,2)\right]^{n-p-1}\left[\frac{1+\eta P_{\text {ex }}}{2}\right]\right\}
\end{aligned}
$$


where the two particle Hamiltonian $\mathrm{H}_{2}$ is equal to

$$
H_{2}(1,2)=\frac{\left[\mathbf{P}_{1}\right]^{2}+\left[\mathbf{P}_{2}\right]^{2}}{2 m}+V(1,2)
$$

Introducing the summation index $p^{\prime}=n-p-1$, we can write

$$
\begin{array}{r}
\mathrm{d} U_{2}^{\mathrm{S}, \mathrm{A}}=\mathrm{d} x \sum_{p, p^{\prime}=0}^{\infty}\left\{\frac{1}{\left[p+p^{\prime}+1\right] !}\left[-\beta H_{2}\right]^{p}\left|1: \varphi_{a} ; 2: \varphi_{b}\right\rangle\left\langle 1: \theta_{a} ; 2: \theta_{b}\right| \times\right. \\
\left.\times\left[-\beta H_{2}\right]^{p^{\prime}}\left[\frac{1+\eta P_{\mathrm{ex}}}{2}\right]\right\}
\end{array}
$$

which, after insertion into (33) and (A.2), gives

$$
\rho_{\mathrm{II}}=\sum_{p, p^{\prime}=0}^{\infty} \frac{1}{\left[p+p^{\prime}+1\right] !}\left[-\beta H_{2}(1,2)\right]^{p}[1+\eta f(1)][1+\eta f(2)]\left[-\beta H_{2}(1,2)\right]^{p^{\prime}}\left[1+\eta P_{\mathrm{ex}}\right]
$$

This result is indeed different from (51) - (53). The brackets $[1+f]$ now appear in the center of the product of operators, instead of at both ends, and there are two of them instead of four. Moreover, series which cannot be summed in exponentials or operators $f$ remain explicitly in the final result. If we insert $V(1,2)=0$ into this expression, we readily recover the expression of the two-body operator given in (29).

We now study the properties of (A.7) at short relative distances and show that the two particle distribution indeed vanishes in regions of the configuration space where the repulsion Is sufficiently strong. Assume that we keep $p^{\prime}$ fixed and sum over $p$. If $p^{\prime}=0$, the sum over $p$ of $\left[-\beta \mathrm{H}_{2}\right]^{p}$ introduces the operator

$$
\frac{\mathrm{e}^{-\beta H_{2}}-1}{\left[-\beta H_{2}\right]}
$$

In the same way, if $p^{\prime}=1$, the same summation introduces

$$
\frac{e^{-\beta H_{2}}-\left[1-\beta H_{2}\right]}{\left[-\beta H_{2}\right]^{2}}
$$

and so on for $p^{\prime}=2,3, \ldots$ for each value of $p^{\prime}$ we obtain a function which contains in the numerator an exponential and a polynomial in $\mathrm{H}_{2}$ with a degree which is inferior to that of the numerator. Therefore, if $\mathrm{H}_{2}$ becomes infinite, all these expressions vanish. Now, assume that we calculate the matrix element

$$
\left\langle\mathbf{r}_{1}, \mathbf{r}_{2}=\mathbf{r}_{1}+\mathbf{r}\left|\rho_{\text {II }}\right| \Psi\right\rangle=\sum_{p^{\prime}}\left\langle\mathbf{r}_{1}, \mathbf{r}_{2}=\mathbf{r}_{1}+\mathbf{r}\left|\sum_{p} \frac{1}{\left[p+p^{\prime}+1\right] !}\left[-\beta H_{2}\right]^{p}\right| \Theta_{p^{\prime}}\right\rangle
$$

where

$$
\left|\Theta_{p^{\prime}}\right\rangle=[1+\eta f(1)][1+\eta f(2)]\left[-\beta H_{2}\right]^{p^{\prime}}|\Psi\rangle
$$

and where $|\Psi\rangle$ is any (fixed) ket. If we insert a closure relation over the eigenstates $\left|\varphi_{q}\right\rangle$ of $H_{2}$, with energies $E_{q}$, we get

$$
\begin{aligned}
\sum_{q}\left\{\left\langle\mathbf{r}_{1}, \mathbf{r}_{2}=\mathbf{r}_{1}+\mathbf{r} \mid \varphi_{q}\right\rangle\left\langle\varphi_{q} \mid \Theta_{0}\right\rangle\left[\frac{e^{-\beta E_{q}}-1}{-\beta E_{q}}\right]+\right. \\
\left.+\left\langle\mathbf{r}_{1}, \mathbf{r}_{2}=\mathbf{r}_{1}+\mathbf{r} \mid \varphi_{q}\right\rangle\left\langle\varphi_{q} \mid \Theta_{1}\right\rangle\left[\frac{e^{-\beta E_{q}}-\left[1-\beta E_{q}\right]}{\left[-\beta E_{q}\right]^{2}}\right]+\left[\text { term } p^{\prime}=2\right]+\text { etc. }\right\}
\end{aligned}
$$


All terms $p^{\prime}=0,1,2, .$. in the right-hand side of this equation contain different fractions which vanish when the energy becomes infinite. Assume now that the interaction potential is strongly repulsive at short relative distances. Then, if $\mathbf{r}$ is sufficiently small, the scalar product $\left\langle\mathbf{r}_{1}, \mathbf{r}_{2}=\right.$ $\mathbf{r}_{1}+\mathbf{r}\left|\varphi_{q}\right\rangle$ will be non zero only if the energy $E_{q}$ is sufficiently large; in the limit of hard core repulsion, it actually completely vanishes if $|\mathbf{r}| \leq b$, where $b$ is the range of the interaction. This shows that all terms in the right hand side of (A.12) become zero at short relative distances $\left({ }^{11}\right)$, and therefore that $\rho_{\mathrm{II}}$ has the cancellation properties discussed in Section 4 . Obviously the same reasoning applies to the conjugate matrix element $\left\langle\Psi\left|\rho_{\mathrm{II}}\right| \mathbf{r}_{1}, \mathbf{r}_{2}=\mathbf{r}_{1}+\mathbf{r}\right\rangle$, provided that the summation over $p^{\prime}$ is now made before the summation over $p\left({ }^{12}\right)$. Equation (A.7) therefore contains the desired non-perturbative cancellation properties of $\rho_{\mathrm{II}}$ at short relative distances. We note that, with this method, the derivative with respect to the potential is first-order instead of second-order; there is a closer relation between the value of $\log Z$ and $\rho_{\mathrm{II}}$, so that the result obtained for the latter could be less sensitive to approximations made in $\log Z$. On the other hand, the big advantage of the method used in the text is that it provides compact analytic expressions where all summations have been incorporated into exponentials or fractions of operators.

\section{Appendix B}

As shown in [2], it is possible to sum an infinite numbers of terms containing powers of $U_{2}$ going to infinity (ladder diagrams, associated with the condensation of pairs) into the following expression

$$
\log Z=[\log Z]_{\mathrm{ig}}-\operatorname{Tr}_{1,2}\left\{\log \left[1-z^{2} U_{2}^{\mathrm{S}, \mathrm{A}}(1,2) \times[1+\eta f(1)] \times[1+\eta f(2)]\right]\right\}
$$

which can be simplified into

$$
\log Z=[\log Z]_{\mathrm{ig}}-\operatorname{Tr}_{1,2}\left\{\log \left[1-z^{2} \widehat{U}_{2}^{\mathrm{S}, \mathrm{A}}(1,2)\right]\right\}
$$

with the notation

$$
\widehat{U}_{2}^{S, A}(1,2)=U_{2}^{\mathrm{S}, \mathrm{A}}(1,2) \times[1+\eta f(1)] \times[1+\eta f(2)] .
$$

But the logarithm of (B.2) can be expanded as

$$
\sum_{n=1}^{\infty} \frac{1}{n} \operatorname{Tr}_{1,2}\left\{\left[\bar{U}_{2}^{\mathrm{S}, \mathrm{A}}(1,2) f(1) f(2)\right]^{n}\right\}
$$

which is a conventent expression for varying the $U_{1}$ 's, and therefore the $f$ 's. At this point, the discussion becomes similar to that given for the ideal gas (by circular permutation under the trace, the variation in $\mathrm{d} x$ can always be brought in front of all the other operators, which eliminates in practice the factor $1 / n$ ) as well as that given after equation (57): the $f$ 's can be expanded as in (35) and the variations of the $U_{1}$ may occur either inside the same $f$, or in two

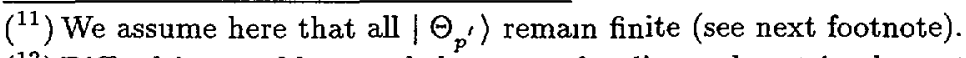

$\left.{ }^{12}\right)$ Difficulties would nevertheless arise for diagonal matrix elements $\left\langle\mathbf{r}_{1}, \mathbf{r}_{2}=\mathbf{r}_{1}+\mathbf{r}\left|\rho_{\mathrm{II}}\right| \mathbf{r}_{1}, \mathbf{r}_{2}=\right.$ $\left.\mathbf{r}_{1}+\mathbf{r}\right\rangle$ with $|\mathbf{r}| \leq b$ : the kets $\left|\Theta_{p^{\prime}}\right\rangle$ would become infinite under the effect of the factor $\left[-\beta H_{12}\right]^{p^{\prime}}$ in (A.11). As in the method used in Section 4, a more detailed study including higher order terms is needed when the particles become close in both the bra and the ket; we do not study this particular case here. 
consecutive $f$ 's, or in two non-consecutive $f$ 's related or not to the same particle. In the first case, $(58)$ is used, and we obtain the following contribution to $\rho_{I I}$

$$
\eta P_{\text {ex. }} B(1,2)+B(1,2) \eta P_{\text {ex. }}
$$

with

$$
B(1,2)=f(1) \otimes[1+\eta f(2)] \operatorname{Tr}_{3}\left\{\frac{z^{2} \widehat{U}_{2}^{S, A}(2,3)}{1-z^{2} \widehat{U}_{2}^{S, A}(2,3)}\right\} .
$$

This is a term which contains no dynamical correlations but just exchange correlations; it is reminiscent of an exchange term for the ideal gas.

If two consecutive $f$ 's are varied, we now use (61) and a simlar calculation gives the following contribution to the two particle density operator $\rho_{\mathrm{II}}$

$$
\begin{aligned}
2 z^{2}[1+\eta f(1)] \times & {[1+\eta f(2)] \frac{1}{1-z^{2} U_{2}^{\mathrm{S}, \mathrm{A}}(1,2)[1+\eta f(1)][1+\eta f(2)]} \times } \\
& \times U_{2}^{\mathrm{S}, \mathrm{A}}(1,2)[1+\eta f(1)][1+\eta f(2)]
\end{aligned}
$$

which can also be written as

$$
2 z^{2}[1+\eta f(1)] \times[1+\eta f(2)] \frac{z^{2} \widehat{U}_{2}^{\mathrm{S}, \mathrm{A}}(1,2)}{1-z^{2} \widehat{U}_{2}^{\mathrm{S}, \mathrm{A}}(1,2)}
$$

This term is the generalization of the first line of (53) and of (62); it contains no trace over extra particles and introduces short-range correlations for strongly repulsive potentials.

The last two terms contain, as in (B.6), a trace over a third particle, which gives them a less important role in the introduction of short-range correlations for hard cores (see the discussion of Section 4); to save space we do not write them explicitly here. So (B.8) is the result of this Appendix.

\section{References}

[1] Grüter P. and Laloë F., J. Phys. France 5 (1995) 181.

[2] Laloë F., Bose Einstein Condensation, A. Griffin, D.W. Snoke and S. Stringarı, Eds. (Cambridge University Press, 1993).

[3] McLennan J.A., Introduction to nonequilibrium statistical mechanics, Chapt. 7, (Prentice Hall, 1989).

[4] Snider R.F., J. Chem. Phys. 32 (1960) 1051.

[5] Bloch C. and de Dommicis C., Nuclear Physics 7 (1958) 459.

[6] Bloch C. and de Dominucis C., Nuclear Physics 10 (1959) 181.

[7] Morice O., Castin Y. and Dalibard J., Phys. Rev. A 51 (1995) 3896.

[8] McLennan J.A., Introduction to nonequilibrium statistical mechanics, Chapt. 8 (Prentice Hall, 1989). 